\title{
FLORÍSTICA Y RELACIONES FITOGEOGRÁFICAS DEL MATORRAL Xerófilo en el Valle de Tecozautla, Hidalgo, México
}

\author{
Sonia Rojas', Carlos Castillejos-Cruz y Eloy Solano \\ Unidad de Investigación en Sistemática Vegetal y Suelo, Carrera de Biólogo, Facultad de Estudios Superiores Zaragoza, \\ Universidad Nacional Autónoma de México, México, D. F., México \\ 'Autor para la correspondencia: xsony@colpos.mx
}

\begin{abstract}
Resumen: Se realizó un estudio florístico y se consideraron las relaciones fitogeográficas del matorral xerófilo en el Valle de Tecozautla, Hidalgo, México. Se inventariaron 479 especies de plantas vasculares, 298 géneros, 80 familias y 51 taxa infraespecíficos. Las familias con mayor número de especies son Asteraceae (62), Poaceae (46) y Fabaceae (33). Las hierbas son la forma de vida dominante. La mayoría de las especies se distribuyen en la zona árida-semiárida. El 37.8\% de las especies se distribuyen en México y el $14.3 \%$ desde el sur de Estados Unidos de América a México. Con base en el coeficiente de similitud de Preston, se comparte el $71 \%$ de los géneros con el Valle de Actopan, 66\% con El Olivo, Ixmiquilpan, ambos localizados en Hidalgo, 54\% con la Sierra de Catorce, San Luis Potosí y 50\% con el Valle de Tehuacán-Cuicatlán, estados de Puebla y Oaxaca. Existe una mayor afinidad con la zona árida del Valle del Mezquital y en general con la porción sur del Desierto Chihuahuense. Se distinguen cinco asociaciones de matorral: Fouqueria splendens-Agave striata, Mimosa-Myrtillocactus, Opuntia, Opuntia imbricata-Myrtillocactus y Yucca. Debido a la falta de endemismos, los elementos florísticos del matorral xerófilo del Valle de Tecozautla probablemente migraron al área en épocas recientes.
\end{abstract}

Palabras clave: Desierto Chihuahuense, Valle del Mezquital, zonas áridas.

\begin{abstract}
We conducted a floristic study and considered phytogeographical relations of the xeric scrubland in Tecozautla Valley, Hidalgo, Mexico. A total of 479 species of vascular plants, 298 genera, 80 families, and 51 infraspecific taxa were found. The most representative families are Asteraceae (62), Poaceae (46), and Fabaceae (33). Herbaceous plants are the dominant life form. Most of species are distributed in arid and semi-arid vegetation types. The $37.8 \%$ of the species were distributed in Mexico and $14.3 \%$ in the southern United States to Mexico. According to the similarity coefficient of Preston, $71 \%$ of the genera is shared with the Actopan Valley, $66 \%$ with El Olivo, Ixmiquilpan, both located in the Hidalgo state, $54 \%$ with the Sierra de Catorce, San Luis Potosi, and $50 \%$ with Tehuacán-Cuicatlán Valley, Puebla and Oaxaca states. The higher affinity was found between the arid zone of Mezquital Valley and the southern portion of the Chihuahuan Desert in general. There are five associations of scrub vegetation: Fouqueria splendens-Agave striata, Mimosa-Myrtillocactus, Opuntia, Opuntia imbricata-Myrtillocactus, and Yucca. Due to lack of endemism, the floristic elements of the desert scrub of Tecozautla Valley, probably migrated to the area in recent times.
\end{abstract}

Key words: arid areas, Chihuahua Desert, Mezquital Valley.

$\mathbf{E}$ 1 territorio nacional abarca una superficie continental e insular de 1,964,375 km², donde más del 50\% corresponde a zonas áridas y semiáridas (Rzedowski, 1991; INEGI, 1997; González, 2012). Estos ecosistemas xerófilos albergan una gran biodiversidad, y conjuntamente con las zonas templadas, húmedas y subhúmedas, hacen de México un país megadiverso. En estas zonas áridas se han inventariado aproximadamente 6,000 especies de angiospermas, de las cuales entre el 50 al 60\% son endémicas (Rzedowski, 1991; Toledo y Ordoñez, 1993; Challenger, 1998). Muchas de las zonas áridas de México han sido poco estudiadas desde un punto de vista florístico y biogeográfico. Entre los trabajos realizados destacan los de Bravo (1936, 1937), Ruíz (1936, 1937), Ramírez (1936, 1937), González-Quintero (1968), González-Medrano y Sánchez-Mejorada (1972), Hiriart y González-Medrano (1983), Rangel-Calderón (1987), Velasco-Santiago y Ojeda-Rivera (1989) y Soriano-Martínez y López-Soto (1994). La mayoría de estos estudios fueron hechos en los Valles de Actopan e Ixmiquilpan, pertenecientes según Rzedowski (1978), a la Región Xerofítica Mexicana, 
provincia florística de la Altiplanicie. Otros estudios se han ejecutado en la zona Árida Queretano-Hidalguense, perteneciente a la provincia florística del Desierto Chihuahuense, la cual destaca por su gran diversidad florística y endemismos (Medellín, 1982). También la porción sur del Desierto Chihuahuense, donde se localiza el Valle de Tecozautla, ha sido señalada por Zamudio-Ruiz (1984) y Zamudio et al. (1992) como un área con alta diversidad. Hernández-Magaña et al. (2012) confirman esta alta diversidad al registrar en un cuadrante de la Zona Árida Queretano-Hidalguense 219 especies, de las cuales 51 son endémicas de esta zona árida y del desierto Chihuahuense.

Por lo que se refiere a los estudios biogeográficos, Rzedowski (1973) analizó las afinidades de las zonas áridas del norte de México y el suroeste de los Estados Unidos de América, concluyó que la región árida de Sonora tiene más elementos tropicales que la de Chihuahua, circunstancia que determina sus diferencias florísticas. La flora del Desierto Chihuahuense es más similar a la del Valle del Mezquital en Hidalgo, pero difiere florísticamente del Valle de Tehucán-Cuicatlán en Puebla y Oaxaca, debido al aislamiento geográfico de éste último (Rzedowski, 1973). Este autor, en general, registró que la flora de las zonas áridas de México tiene una mayor afinidad con elementos meridionales más que con boreales, e indicó que este resultado evidencia que la flora xerofítica mexicana se originó en su mayor parte de manera independiente de la del suroeste de los Estados Unidos de América. Morrone (2005) menciona que tres de los componentes bióticos que predominan en las zonas áridas subtropicales del norte de México son el Neártico, con elementos septentrionales antiguos o paleoamericanos; otro de dispersión más reciente, y uno más neotropical antiguo.

Las zonas áridas de México tradicionalmente han sido proveedoras de recursos vegetales importantes. González (2012) hace un análisis de las zonas áridas y semiáridas de México, donde menciona la importancia de los recursos bióticos y la interacción que guardan con los grupos étnicos de estas zonas. Sin embargo, el aprovechamiento inadecuado y el tráfico ilegal de especies han ocasionado un grave deterioro de estos ecosistemas, que se manifiesta principalmente con la pérdida de la biodiversidad y erosión de los suelos, procesos naturales que promueven la marginación de poblaciones humanas (Toledo y Ordoñez, 1993). Ante esta problemática es indispensable completar el inventario florístico de México, sobretodo atender zonas perturbadas por actividades antrópicas, como el centro del país (Rzedowski de Calderón y Rzedowski, 1991). Los inventarios contribuyen al conocimiento de la flora nacional y son la base para el manejo adecuado de los recursos vegetales y la realización de estudios fitogeográficos. Además, proporcionan información acerca de áreas de endemicidad, sobre especies incluidas en alguna categoría de riesgo y permiten evaluar áreas protegidas en función de la riqueza y unicidad de su flora (Rzedowski, 1978). En particular el Valle de Tecozautla, Hi- dalgo, presenta una fuerte presión sobre su cubierta vegetal, originada por actividades agrícolas y pecuarias; por lo tanto, el objetivo de este trabajo fue inventariar su flora vascular y determinar las afinidades fitogeográficas de la misma.

\section{Materiales y métodos}

Área de estudio. El Valle se localiza principalmente en el municipio de Tecozautla, al oeste del estado de Hidalgo, aunque también incluye una pequeña parte del municipio de Huichapán; en Querétaro abarca una porción en Ezequiel Montes y Cadereyta. El área se ubica entre los $20^{\circ} 30^{\prime}$ y $20^{\circ}$ $35^{\prime}$ de latitud norte y los $99^{\circ} 30^{\prime}$ y $99^{\circ} 42^{\prime}$ de longitud oeste. Cubre una superficie aproximada de $393 \mathrm{~km}^{2}$ (Figura 1). Limita al norte con la presa Zimapán, al sur con los cerros La Joya y La Petaca, al oeste con La Cruz y San Pedro y al este con El Epazote (INEGI, 2002). En el Valle predominan rocas volcánicas como brechas, tobas, riolitas y basaltos del Terciario y Cuaternario. Tiene una altitud promedio de $1,700 \mathrm{~m}$, con una topografía principalmente plana que cubre el $50 \%$ de su superficie, la otra porción es accidentada (INEGI, 1991). La zona de estudio pertenece a la Región Hidrológica Río Pánuco y el Río Moctezuma es uno de sus afluentes, mismo que sirve de límite natural entre Querétaro e Hidalgo, donde está ubicada la zona de estudio.

El clima predominante, de acuerdo con García (1973), es seco estepario, BS1 hw (w)(e)g, con lluvias en verano e invierno fresco, temperatura media anual de $19.3{ }^{\circ} \mathrm{C}$, precipitación media anual de 500 mm (INEGI, 1982). Los suelos dominantes son feozem háplico y calcárico, xerosol háplico con litosol y regosol éutrico (Consejo Estatal de Ecología. Estado de Hidalgo, 2001). Con base en Rzedowski (1978), la vegetación se clasificó como matorral xerófilo.

El Valle de Tecozautla se delimitó con base en la localización de formaciones geológicas de más de 2,000 m de altura. Se realizaron recorridos de campo para recolectar muestras botánicas, se registraron cerca de 1,000 números y se acumularon 2,376 ejemplares. Los especímenes recolectados se determinaron taxonómicamente con el uso de bibliografía especializada, posteriormente se cotejaron y en algunos casos se consultaron especialistas. Un duplicado de estos especímenes se depositó en las colecciones FEZA y MEXU.

En el listado florístico, las familias botánicas se organizaron de acuerdo con los siguientes autores: pteridofitas, Mickel y Smith (2004); gimnospermas, McVaugh (1992) y monocotiledóneas y eudicotiledóneas, APG III (2009). La ortografía correcta de los nombres científicos se validó con la base de datos proporcionada por Tropicos del Missouri Botanical Garden (2012). Las autoridades de los taxa se citaron conforme a Brummitt y Powell (1992) y Villaseñor (2001).

La riqueza estimada fue calculada con base en el cociente especies/género, propuesto por Rzedowski (1991), y se comparó con tres regiones: Barranca de Tolantongo, Hidal- 


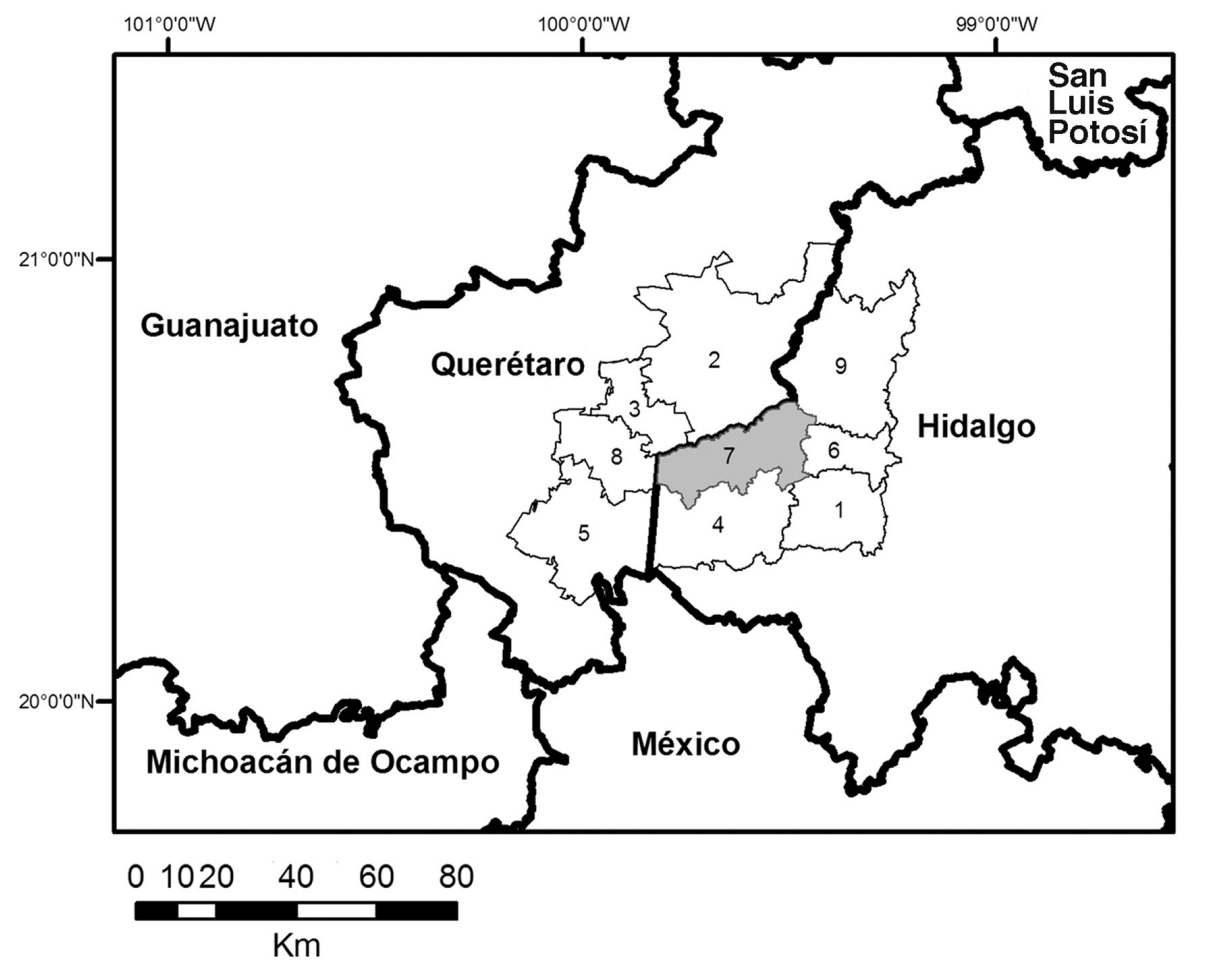

1. Alfajayucan

2. Cadereyta de Montes

3. Ezequiel Montes

4. Huichapan

5. San Juan del Río

6. Tasquillo

7. Tecozautla

8. Tequisquiapan

9. Zimapán

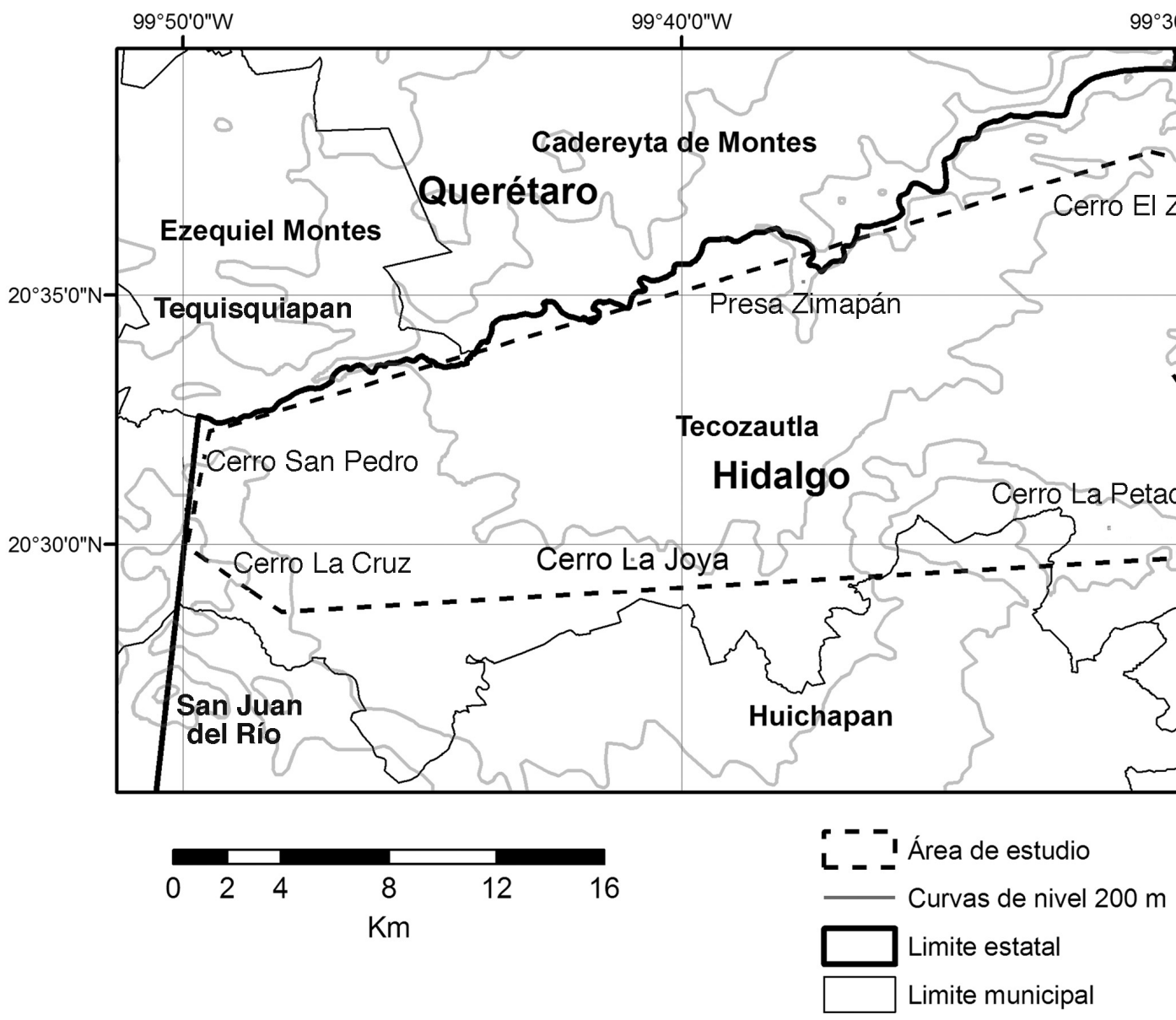

Figura 1. Localización del Valle de Tecozautla, Hidalgo. 
go, (Hiriart y González-Medrano, 1983), Valle de Actopan, Hidalgo (Soriano-Martínez y López-Soto, 1994) y Valle de Santiago, Guanajuato (Aguilera-Gómez, 1991). Por medio del coeficiente de Preston (1962), en el nivel genérico, se estableció la similitud florística con las áreas anteriormente señaladas, además de las regiones El Olivo, Ixmiquilpan, Hidalgo (Sánchez-Sánchez, 2012) y el Valle de TehuacánCuicatlán (Valiente-Banuet et al., 2009), mediante la siguiente fórmula:

$$
\mathrm{T}=\left(\mathrm{A}^{1 / 0.27}+\mathrm{B}^{1 / 0.27}\right)^{0.27}
$$

Donde: $\mathrm{A}$ y $\mathrm{B}$ = número de géneros totales de cada zona estudiada, $\mathrm{T}=$ número teórico total de géneros representados en ambas zonas, si A y B son miembros de la misma flora. Para las mismas condiciones teóricas, el número de géneros comunes en $\mathrm{A}$ y $\mathrm{B}$ son:

$$
\mathrm{K}=\mathrm{A}+\mathrm{B}-\mathrm{T}
$$

y el coeficiente de similitud entre A y B, expresado en porcentaje es:

$$
\mathrm{CS}=100 \mathrm{~L} / \mathrm{K}
$$

donde $\mathrm{L}$ es el número real de géneros comunes entre $\mathrm{A}$ y $\mathrm{B}$.

Las especies inventariadas en el matorral xerófilo del Valle de Tecozautla se ubicaron en los diez tipos de vegetación de Rzedowski (1978). Estos tipos de vegetación se agruparon en cuatro zonas: tropical, templada, árida-semiárida y acuática-subacuática. Asimismo, se consideraron las especies compartidas entre las cuatro áreas mencionadas y se determinaron las formas biológicas de acuerdo con la clasificación de Shreve y Wiggins (1964).

Con base en su distribución geográfica, cada especie se ubicó en alguno de los siguientes patrones: endémicas de México, sur de Estados Unidos de América a México, sur de Estados Unidos a Centroamérica y cosmopolita. Las especies claramente introducidas o cultivadas se excluyeron de este análisis.

\section{Resultados}

Florística. Se registraron 80 familias, 298 géneros, 479 especies y 51 taxa infraespecíficos de plantas vasculares (Apéndice). El promedio de especies por género fue de 1.6 y por familia de 5.9. El mayor número de familias correspondió a las eudicotiledóneas, seguidas de las monocotiledóneas (Cuadro 1). En las angiospermas se ubicaron 94.1\% de las especies, 5.2\% correspondieron a las monilofitas. Las gimnospermas representaron el $0.4 \%$ y las licofitas el $0.2 \%$ de la flora total. Asteraceae, Poaceae y Fabaceae, fueron las familias mejor representadas en el nivel de especie. Los géneros más diversos fueron Cheilanthes, Muhlenbergia, Sedum y Sida (Cuadro 2). De acuerdo con Shreve y Wiggins (1964), la forma biológica predominante son las hierbas, seguidas de los arbustos (Cuadro 3).
Cuadro 1. Riqueza de plantas vasculares en el Valle de Tecozautla, Hidalgo.

\begin{tabular}{lcccc}
\hline & Familias & Géneros & Especies & $\begin{array}{c}\text { Taxa } \\
\text { infraespecíficos }\end{array}$ \\
\hline Licofitas & 1 & 1 & 1 & 0 \\
Monilofitas & 8 & 10 & 25 & 2 \\
Gimnospermas & 1 & 2 & 2 & 1 \\
Monocotiledóneas & 8 & 49 & 77 & 3 \\
Eudicotiledóneas & 62 & 236 & 374 & 45 \\
Total & 80 & 298 & 479 & 51 \\
\hline
\end{tabular}

Cuadro 2. Familias y géneros de plantas vasculares mejor representados en el Valle de Tecozautla, Hidalgo.

\begin{tabular}{cccc}
\hline Familias & Especies & Géneros & Especies \\
\hline Asteraceae & 62 & Cheilanthes & 14 \\
Poaceae & 46 & Muhlenbergia & 7 \\
Fabaceae & 33 & Sedum & 7 \\
Cactaceae & 27 & Sida & 7 \\
Malvaceae & 24 & & \\
\hline
\end{tabular}

Cuadro 3. Formas biológicas de acuerdo con Shreve y Wiggins (1964), presentes en el Valle de Tecozautla, Hidalgo.

\begin{tabular}{ccc}
\hline Formas de vida & \multicolumn{2}{c}{ Especies } \\
\cline { 2 - 3 } & Cantidad & $\begin{array}{r}\text { \% con respecto } \\
\text { a la flora total }\end{array}$ \\
\hline Hierbas (anuales & 277 & 57.8 \\
y perennes) & & \\
Arbustos & 127 & 26.5 \\
Árboles & 35 & 7.3 \\
Trepadoras & 16 & 3.3 \\
Suculentas & 16 & 3.3 \\
Arrosetadas & 5 & 1.0 \\
Parásitas & 3 & 0.6 \\
\hline
\end{tabular}

El cociente especies/género para el total de la flora fue de 1.5, mientras que, para la familia Asteraceae resultó de 1.3; por lo tanto, al multiplicar el cociente de la flora total con el número de géneros totales, la riqueza florística estimada del Valle es de aproximadamente 429, cantidad cercana al número de especies inventariadas. En la familia Asteraceae, la riqueza estimada registro 62 especies, cantidad cercana a la observada (Cuadro 4).

De acuerdo con el coeficiente de Preston (1962), en el nivel genérico, la flora del Valle de Tecozautla comparte el $70.9 \%$ de los géneros con el Valle de Actopan y con la Barranca de Tolantongo, Hidalgo el 43\% de los mismos (Cuadro 5). 297 (66.6\%) especies son exclusivas de la zona ári- 
Cuadro 4. Proporción entre el número de especies y géneros calculado para la familia Asteraceae y algunas regiones geográficas cercanas (cociente e/g).

\begin{tabular}{|c|c|c|c|c|c|c|}
\hline & \multicolumn{3}{|c|}{ Flora fanerogámica total } & \multicolumn{3}{|c|}{ Asteraceae } \\
\hline & Géneros & Especies & $\mathrm{e} / \mathrm{g}$ & Géneros & Especies & $\mathrm{e} / \mathrm{g}$ \\
\hline Barranca de Tolantongo & 225 & 280 & 1.2 & 23 & 26 & 1.1 \\
\hline Valle de Actopan & 187 & 269 & 1.4 & 41 & 54 & 1.3 \\
\hline Valle de Santiago & 175 & 228 & 1.3 & 22 & 23 & 1.0 \\
\hline Valle de Tecozautla & 287 & 453 & 1.5 & 46 & 62 & 1.3 \\
\hline
\end{tabular}

Cuadro 5. Similitudes florísticas entre el Valle de Tecozautla y otras regiones geográficas.

\begin{tabular}{lcccccc}
\hline & A & B & C & D & E & F \\
\hline Número de géneros & 298 & 225 & 188 & 187 & 175 & 280 \\
Géneros comunes & & 92 & 116 & 123 & 99 & 115 \\
Coeficiente de Preston (CS) & & 43.0 & 66.6 & 70.9 & 60.2 & 50.2 \\
\hline
\end{tabular}

$\mathrm{A}=$ Valle de Tecozautla, $\mathrm{B}=$ Barranca de Tolantongo, $\mathrm{C}=$ El Olivo, $\mathrm{D}=$ Valle de Actopan, $\mathrm{E}=$ Valle de Santiago, $\mathrm{F}=$ Valle de TehuacánCuicatlán.

Cuadro 6. Zonas de distribución de las especies de plantas vasculares presentes en el Valle de Tecozautla, Hidalgo, con base en Rzedowski (1978).

\begin{tabular}{lc}
\hline \multicolumn{1}{c}{ Zona } & Número de especies (\%) \\
\hline $\begin{array}{l}\text { Tropical (bosque tropical perennifolio, } \\
\text { bosque tropical subperennifolio, } \\
\text { bosque tropical perennifolio) }\end{array}$ & $0(0)$ \\
Templada (bosque de coníferas, & \\
bosque de Quercus, bosque mesófilo & $20(4.5)$ \\
de montaña) & \\
Árida-semiárida (bosque espinoso, \\
bosque tropical caducifolio, matorral \\
xerófilo, pastizal)
\end{tabular}

da-semiárida, 20 (4.5\%) de la templada y 111 (24.9\%) son comunes para ambas zonas (Cuadro 6, Apéndice).

Distribución geográfica. Este análisis incluyó 446 especies. Se excluyeron 33 por ser claramente introducidas o cultivadas (Apéndice). El patrón con mayor número de especies fue endémicas de México con 169. Dentro de dicho patrón se registraron 53 propias del centro del país y una referida a la zona Árida Queretano-Hidalguense; 53 se distribuyen del norte al centro de México, las cuales están incluidas en el desierto Chihuahuense. En los otros patrones se agruparon
Cuadro 7. Distribución geográfica de las especies presentes en el Valle de Tecozautla, Hidalgo.

\begin{tabular}{lc}
\hline \multicolumn{1}{c}{ Patrón de distribución } & Número de especies \\
\hline Endémicas de México & $169(37.8 \%)$ \\
Sur de Estados Unidos de América a México & $64(14.3 \%)$ \\
Sur de Estados Unidos a Centroamérica & $109(24.4 \%)$ \\
Cosmopolita & $104(23.3 \%)$ \\
\hline
\end{tabular}

un número menor de especies (Cuadro 7). Las taxa muestran afinidad neotropical y destacan elementos florísticos propios de México.

\section{Discusión}

Según Rzedowski (1991), en México se han registrado 220 familias de fanerógamas, y para el estado de Hidalgo, Villavicencio et al. (1998) indicaron 177, mientras que Villaseñor (2003) consideró 175 . Al comparar estas cifras con las obtenidas en este estudio, se puede observar que las 81 familias inventariadas, representan poco más de un tercio de las fanerógamas del país y casi el 50\% para el estado de Hidalgo, de donde se deduce que a pesar de ser un área pequeña contiene una alta diversidad florística, como ya había sido señalado por Medellín (1982) y Zamudio et al. (1992); sin embargo, las gimnospermas y las licofitas están pobremente representadas $(0.6 \%)$.

De acuerdo con Rzedowski (1991), las especies de la familia Asteraceae son abundantes en las regiones montañosas y las zonas áridas y semiáridas de México; además, están mejor representadas en el norte y el centro del país; quizá por esta razón, Asteraceae es la más diversa en la zona estudiada. Enseguida se ubicó Poaceae, familia que ocupa el tercer lugar en el mundo en cuanto a diversidad de especies. Para México, Dávila et al. (2006) señalaron que existen 207 géneros y 1,182 especies; por lo tanto, los 29 géneros y las 46 especies registradas en la zona de estudio, representan el $14 \%$ y el 3.9\% respectivamente del total de las gramíneas de México. Estos valores mínimos probablemente se deben a que las gramíneas se distribuyen en zonas perturbadas, comportándose generalmente como ruderales o arvenses, y existe poca diversidad de ellas en el matorral conservado. Fabaceae es la tercera familia mejor representada en el Valle de Tecozautla con un 6.8\%. Sousa y Delgado (1993) documentaron que esta familia ocupa el segundo lugar en diversidad de especies en México y que éstas se encuentran ampliamente distribuidas en todo el territorio nacional; además, sus especies arbustivas y herbáceas son elementos típicos de los matorrales xerófilos mexicanos (Rojas-Mendoza, 1965; Rzedowski, 1978; Briones y Villarreal, 2001).

Los géneros con mayor número de especies corresponden a taxa con alta diversidad en el país. Así, Cheilanthes es el más diverso en la zona de estudio, con 14 especies 
(2.91\%); de acuerdo con Mickel y Smith (2004), en México existen 150 especies de este género, de éstas, casi la mitad están adaptadas a las condiciones ecológicas del matorral xerófilo. Enseguida se encuentra Muhlenbergia, Sedum y Sida, con siete especies respectivamente. En la República Mexicana, el primer género consta de 120 especies y es uno de los más diversos de la familia Poaceae (Herrera y Cortés, 2009). El territorio nacional se considera un centro de diversificación del género Sedum, donde se han registrado 110 especies. Sida es un género de aproximadamente 150 especies nativas de América, África, Asia y Australia, 36 de ellas se encuentran en la República Mexicana (Fryxell, 1988, 1993).

En el área de estudio la dominancia de herbáceas y arbustos se relaciona con el clima seco y los suelos pedregosos y superficiales, donde estas formas de vida han desarrollado diversas adaptaciones, entre ellas, sistemas radicales extendidos, hojas pequeñas o reducidas a espinas, pubescencia y suculencia, entre otras (Whittaker, 1975). En las escasas cañadas con mayor humedad, baja intensidad lumínica o pendientes pronunciadas, se forman microhábitats que favorecen el establecimiento de formas arbóreas como Celtis reticulata, Salix taxifolia, Sapindus saponaria y Zanthoxilum fagara, principalmente.

En el Valle de Tecozautla se distinguen cinco asociaciones de matorral: Fouquieria splendens-Agave striata, MimosaMyrtillocactus, Opuntia, Opuntia imbricata-Myrtillocactus y Yucca. La primera asociación se establece principalmente en pendientes y laderas de cerros a 2,000 m aproximadamente, en amplias extensiones con suelos derivados de material calcáreo. Los elementos florísticos predominantes son Fouquieria splendens y diferentes especies del género Agave. En el estrato herbáceo se encuentran Carlowrightia lindauiana, Melinis repens, Plumbago pulchella, Schkuhria pinnata, Selaginella lepidophylla y Zinnia peruviana, como epifita se encuentra Tillandsia recurvata. Fouquieria splendens forma un estrato arbustivo bastante denso de 1.5 a 3 $\mathrm{m}$ de alto, junto con Condalia mexicana, Jatropha dioica y Mimosa biuncifera; son frecuentes Agave lechuguilla y A. striata, además de Echinocereus cinerascens y Karwinskia humboldtiana.

La asociación Mimosa-Myrtillocactus se localiza en zonas planas poco profundas, entre 1,900 a 2,200 m, en las áreas más secas, sobre suelos pedregosos pardo-oscuros. Se caracteriza por la presencia de un estrato herbáceo conformado por Erigeron pubescens, Tridax coronopifolia y algunas plantas postradas como Evolvulus prostratus y Nama dichotomun; además de plantas trepadoras, entre ellas Ipomoea orizabensis, I. purpurea y Matelea sp., y como epifita se encuentra Tillandsia recurvata; el estrato arbustivo es de 1.5 a $2.5 \mathrm{~m}$ de alto y está constituido por Condalia mexicana, Fouquieria splendens, Jatropha dioica, Mimosa depauperata y Opuntia joconostle; también se desarrolla un estrato arbóreo y formas arborescentes que alcanza de 3 a 5 m de alto, constituido por Bursera fagaroides, Myrtillocactus geometrizans, Stenocerus dumortieri y Yucca filifera.

El matorral de Opuntia se encuentra en zonas planas y laderas de cerros con poca pendiente, entre los 1,700 y 2,100 $\mathrm{m}$, sobre suelos someros, pardos. En el estrato herbáceo se presentan Bouteloua curtipendula, Lasianthaea aurea y Zinnia peruviana; mientras que el arbustivo alcanza una altura de 1 a $2 \mathrm{~m}$ y está constituido principalmente por especies del género Opuntia, tales como $O$. durangensis, $O$. microdasys y O. tomentosa; también participan Ageratina hidalguensis, Mimosa biuncifera y Zaluzania augusta.

La asociación Opuntia imbricata-Myrtillocactus se localiza a $1,900 \mathrm{~m}$. Es un matorral abierto, sobre laderas de cerros y zonas con poca inclinación, con una altura de 1.5 a $2 \mathrm{~m}$ y se desarrolla sobre suelos someros, pardo-oscuros. El estrato herbáceo está representado por Asclepias linaria, Commelina erecta, Erigeron pubescens, Euphorbia antisyphillitica, Herissantia crispa, Hibiscus elegans, entre otras. En el estrato arbustivo domina Opuntia imbricata con otros elementos como Ditaxis heterantha, Karwinskia humboldtiana y Lantana hirta. Destaca Myrtillocactus geometrizans como elemento arborescente.

El matorral de Yucca se desarrolla entre 1,800 a 2,300 m, en suelos aluviales profundos y planos o con poca pendiente. El estrato herbáceo es muy escaso y en él destacan Agave americana, Evolvulus prostratus, Rhynchosia prostrata y Sanvitalia procumbens. El estrato arbustivo es bajo (30 a 60 $\mathrm{cm})$ y en él se pueden encontrar Chrysactinia mexicana, Dalea versicolor, Dyssodia acerosa y Opuntia durangensis.

La flora de la zona de estudio en el nivel genérico tiene mayor similitud con la del Valle de Actopan y la región de El Olivo, ambas localizadas en el Valle del Mezquital, Hidalgo, estas áreas pertenecen al Desierto Chihuahuense donde también se ubica la zona de estudio. Comparte menos géneros con la flora del Valle de Tehucán-Cuicatlán, seguida de La Sierra de Catorce, San Luis Potosí y finalmente el Valle de Santiago, Guanajuato. Rzedowski (1973) al analizar las similitudes florísticas de las zonas áridas y semiáridas del norte de México y el suroeste de Estados Unidos de Norteamérica, encontró patrones de similitud semejantes.Tanto en el Valle de Tecozautla como en las zonas áridas del norte de México se desarrolla el matorral xerófilo, sobre suelos calcáreos pobres en materia orgánica.

En el Valle de Tecozautla, más del 90\% del total de la flora inventariada se distribuye en las zonas árida-semiárida y templada. El $66.44 \%$ de las especies habitan exclusivamente en el primer tipo y el $25 \%$ son comunes en ambos tipos. Rzedowski (1978) mencionó que la vegetación de la zona árida y semiárida cubre la mayor superficie de México y contribuye con aproximadamente $45 \%$ de las especies a la flora total (Rzedowski, 1991). Un porcentaje mínimo de especies (2.6\%), entre ellas Cheilanthes lendigera, Cyperus hermaphroditus, Metastelma mexicanum y Tithonia tubiformis, se distribuyen tanto en la zona tropical, templada, 
árida-semiárida y acuática-subacuática. Según Naoki et al. (2006), las especies de amplia distribución ecológica pueden tolerar condiciones climáticas muy variables y distribuirse en varios tipos de vegetación.

En general, las especies inventariadas tienen afinidad meridional. Además, en la zona estudiada ocurren taxa endémicos típicos del centro de México. En este contexto, Rzedowski (1978) indicó que en la flora de las zonas áridas mexicanas, las afinidades meridionales dominan ampliamente sobre las boreales y que los elementos predominantes de la vegetación xerofítica son el neotropical y el endémico; mientras que, la influencia de la flora holártica es menos importante. En la flora del Valle de Tecozautla están presentes especies provenientes de las partes áridas y semiáridas del sur de México, como Coreopsis mutica, Heliotropium pringlei e Ipomoea murocoides, entre otras. Asimismo, el Valle comparte especies con las regiones áridas del norte, entre ellas, Agave lechuguilla, Cheilanthes pringlei, Juniperus flaccida y Selaginella lepidophylla, y con el sur de América Bouteloa curtipendula, Commelina diffusa y Tinantia erec$t a$, que probablemente se originaron en Sudamérica a inicios del Terciario (Johnston, 1940). Por otro lado, según Axelrod $(1948,1950)$, el origen de la flora boreal y meridional de Norteamérica es diferente. La primera es característica del desierto de la Gran Cuenca y pudo haberse originado en el Terciario a partir de bosques de coníferas. La meridional se origina en los desiertos de Sonora y Mohave, probablemente de una flora tropical similar a la que evolucionó en Sudamérica y en todos los desiertos cálidos, esto último podría explicar la afinidad que existe entre los desiertos Sonorense y Chihuahuense con los desiertos sudamericanos (Delgadillo-Rodríguez y Macías-Rodríguez, 2002).

En el nivel genérico domina la afinidad neotropical. Según Challenger (1998), parte de Hidalgo se incluye en el Reino Holártico y el valle estudiado se localiza en los límites de éste y el Neotropical. Es probable que los géneros del matorral estudiado se hayan originado a partir de elementos florísticos neotropicales, pero actualmente incluye géneros comunes con las regiones del norte de México y el suroeste de Estados Unidos de América; esta última región según Villaseñor et al. (1990), juega un papel importante en los patrones de distribución de muchos componentes de la flora mexicana.

Es factible que la flora del Valle de Tecozautla arribó recientemente y está conformada por linajes muy antiguos que posiblemente se originaron en los desiertos norteamericanos, principalmente el Chihuahuense. Sin embargo, es probable que este matorral, junto con otros del centro de México, formen parte de la geoflora Madro-Terciaria (Axelrod, 1958), la cual constituyó un cinturón de vegetación mundial que se dispersó desde los bordes de los trópicos norteamericanos a partir del Eoceno Medio, como resultado de la expansión del clima árido en Norteamérica (García et al., 1960; Rzedowski, 1993; González-Medrano, 1998; Valiente-Banuet et al., 1998; Valiente-Banuet et al., 2000).

\section{Agradecimientos}

Se agradece a J. Rzedowski y a José Luís Villaseñor por sus valiosas sugerencias, así como a dos revisores anónimos por sus observaciones al manuscrito original. Abisaí J. García Mendoza, y J. L. Villaseñor, revisaron algunos ejemplares de las familias Agavaceae y Asteraceae. Jaime Santiago Mariscal preparó el mapa de la región.

\section{Literatura citada}

Aguilera-Gómez J.L. 1991. Estudio florístico y sinecológico de la vegetación en el cráter "Hoya de Rincón de Paranguero", Valle de Santiago, Gto. Tesis de maestría, Colegio de Postgraduados, Montecillo, Estado de México. 99 pp.

APG III (The Angiosperm Phylogeny Group). 2009. An update of the Angiosperm Phylogeny Group classification for the orders and families of flowering plants: APG III. Botanical Journal of the Linnean Society 161:105-121.

Axelrod D.I. 1948. Climate and evolution in western North America during Middle Pliocene time. Evolution 2:127-144.

Axelrod D.I. 1950. Evolution of desert vegetation in western North America. Carnegie Institute Washington 590:215-306.

Axelrod D.I. 1958. Evolution of the Madro-Tertiary Geoflora. The Botanical Review 24:433-509.

Bravo H. 1936. Observaciones florísticas y geobotánicas en el Valle de Actopan. Anales del Instituto de Biología de la Universidad Nacional Autónoma de México 7:169-233.

Bravo H. 1937. Observaciones florísticas y geobotánicas en el Valle del Mezquital. Anales del Instituto de Biología de la Universidad Nacional Autónoma de México 8:3-82.

Briones O. y Villarreal Q.J.A. 2001. Vegetación y flora de un ecotono entre las provincias del Altiplano y de la Planicie Costera del Noreste de México. Acta Botanica Mexicana 55:39-67.

Brummitt R.K. y Powell C.E. 1992. Authors of Plant Names. Royal Botanic Gardens, Kew.

Challenger A. 1998. Utilización y Conservación de los Ecosistemas Terrestres de México. Pasado, Presente y Futuro. Comisión Nacional para el Conocimiento y Uso de la Biodiversidad-Instituto de Biología, Universidad Nacional Autónoma de México. México, D.F.

Consejo Estatal de Ecología. Estado de Hidalgo. 2001. Síntesis del Ordenamiento Ecológico Territorial del estado de Hidalgo. Secretaría del Medio Ambiente y Recursos Naturales. México, D.F.

Dávila P.A., Mejía-Saulés M.T., Gómez S.M., Valdés R.J., Ortiz J.J., Morín C., Castrejón J. y Ocampo A. 2006. Catálogo de las Gramíneas de México. Universidad Nacional Autónoma de México-Comisión Nacional para el Conocimiento y Uso de la Biodiversidad. México, D.F.

Delgadillo-Rodríguez J. y Macías-Rodríguez M.A. 2002. Componente florístico del desierto de San Felipe, Baja California, México. Boletín de la Sociedad Botánica de México 70:45-65.

Fryxell P.A. 1988. Malvaceae of Mexico. Systematic Botany Monographs 25:1-522.

Fryxell P.A. 1993. Malvaceae. En: Rzedowski J. y Calderón de Rzedowski G. Eds. Flora del Bajío y de Regiones Adyacentes, pp. 1-174, fascículo 16, Instituto de Ecología A.C. Centro Regional del Bajío, Consejo Nacional de Ciencia y Tecnología, 
Comisión Nacional para el Conocimiento y Uso de la Biodiversidad, Pátzcuaro.

García E. 1973. Modificaciones al Sistema de Clasificación Climática de Köppen. Instituto de Geografía, Universidad Nacional Autónoma de México, México, D.F.

García E., Soto C. y Miranda F. 1960. Larrea y clima. Anales del Instituto de Biología de la Universidad Nacional Autónoma de México 31:133-171.

González M.F. 2012. Las Zonas Áridas y Semiáridas de México y su Vegetación. Secretaría de Medio Ambiente y Recursos Naturales, Instituto Nacional de Ecología. México, D.F.

González-Medrano F. 1998. La vegetación de México y su historia. Ciencias 52:58-65.

González-Medrano F. y Sánchez-Mejorada H. 1972. Excursión a la Barranca de Meztitlán, Hidalgo. En: Sociedad Botánica de México Ed. Guías Botánicas de Excursiones en México, pp. 6368, México, D.F.

González-Quintero L. 1968. Tipos de Vegetación del Valle del Mezquital, Hidalgo. Departamento de Prehistoria, Instituto Nacional de Antropología e Historia. México, D.F.

Herrera A.Y. y Cortés O.A. 2009. Diversidad de las gramíneas de Durango, México. Polibotánica 28:49-68.

Hernández-Magaña R., Hernández-Oria J.G. y Chávez R. 2012. Datos para la conservación florística en función de la amplitud geográfica de las especies en el Semidesierto Queretano, México. Acta Botanica Mexicana 99:105-140.

Hiriart V.P. y González-Medrano F. 1983. Vegetación y fitogeografía de la Barranca de Tolantongo, Hidalgo, México. Anales del Instituto de Biología de la Universidad Nacional Autónoma de México, Serie Botánica 54:29-96.

INEGI. Instituto Nacional de Estadística, Geografía e Informática. 1982. Carta climatológica. Hoja Pachuca. Esc. 1:1 000000. Sistema meteorológico mexicano. México, D.F.

INEGI. Instituto Nacional de Estadística, Geografía e Informática. 1991. Carta topográfica. Hoja Pachuca. Esc. 1:250 000. Sistema meteorológico mexicano. México, D.F.

INEGI. Instituto Nacional de Estadística, Geografía e Informática. 1997. Carta de uso del suelo y vegetación. Hoja Pachuca. Esc. 1:1 000 000. Sistema meteorológico mexicano. México, D.F.

INEGI. Instituto Nacional de Estadística, Geografía e Informática. 2002. Marco geoestadístico municipal 2000. México, D.F.

Johnston I.M. 1940. The floristic significance of shrubs common to north and south American deserts. Journal of the Arnold Arboretum 21:356-363.

McVaugh R. 1992. Gymnosperms and Pteridophytes. En: Anderson W.R. Ed. Flora Novo-Galiciana. Vol. 17, pp. 1-467, The University of Michigan Press, Ann Harbor.

Medellín F. 1982. The Chihuahua desert. En: Bender G.L. Ed. Reference Handbook on the Deserts of North America, pp. 321381, Greenwood Press, Westport.

Mickel J.T. y Smith A.R. 2004. The Pteridophytes of Mexico. Part I. The New York Botanical Garden Press, Nueva York.

Mickel J.T. y Smith A.R. 2004. The Pteridophytes of Mexico. Part II. The New York Botanical Garden Press, Nueva York.

Morrone J.J. 2005. Hacia una síntesis biográfica de México. Revista Mexicana de Biodiversidad 76:207-252.

Naoki K., Gómez M.I., López R.P., Meneses R.I. y Vargas J. 2006. Comparación de modelos de distribución de especies para predecir la distribución potencial de vida silvestre en Bolivia. Ecología en Bolivia 41:65-78.
Preston F.W. 1962. The canonical distribution of commonness and rarity: Part I. Ecology 43:185-215.

Ramírez L.A. 1936. Contribución al conocimiento de los agaves del Valle de Actopan, Hgo. Anales del Instituto de Biología Universidad Nacional Autónoma de México 7:235-240.

Ramírez L.A. 1937. Notas acerca del aprovechamiento de algunas plantas de importancia económica en la región del Valle del Mezquital. Anales del Instituto de Biología Universidad Nacional Autónoma de México 8:83-115.

Rangel-Calderón S. 1987. Etnobotánica de los agaves del Valle del Mezquital. Tesis de licenciatura, Escuela Nacional de Estudios Profesionales Iztacala, Universidad Nacional Autónoma de México. México, D.F. 155 pp.

Rojas-Mendoza P. 1965. Generalidades sobre la vegetación del estado de Nuevo León y datos acerca de su flora. Tesis doctoral, Facultad de Ciencias, Universidad Nacional Autónoma de México, México, D.F., 124 pp.

Ruíz O.M. 1936. Contribución al conocimiento de los líquenes del Valle de Actopan. Anales del Instituto de Biología de la Universidad Nacional Autónoma de México 7:241-249.

Ruíz O.M. 1937. Contribución al conocimiento de los líquenes del Valle del Mezquital. Anales del Instituto de Biología de la Universidad Nacional Autónoma de México 8:117-131.

Rzedowski J. 1973. Geographical relationships of the flora of Mexican dry regions. En: Graham A. Ed. Vegetation and Vegetational History of Northern Latin America, pp. 61-72, Elsevier Science Publication Company, Amsterdam.

Rzedowski J. 1978. Vegetación de México. Limusa. México, D.F.

Rzedowski J. 1991. Diversidad y orígenes de la flora fanerogámica de México. Acta Botanica Mexicana 14:3-21.

Rzedowski J. 1993. Reflexiones y experiencias sobre el trabajo florístico en México. En: Herbario Nacional Forestal, Instituto Nacional de Investigaciones Forestales y Agropecuarias, Eds. Memorias del XXX Aniversario del Herbario Nacional Forestal $y$ de la VII Reunión Nacional de Encargados de Herbario, pp. 49-58, Publicación Especial, México, D.F.

Rzedowski de Calderón G. y Rzedowski J. 1991. Presentación. Guía para los autores y normas editoriales. Flora del Bajío y de Regiones Adyacentes. Fascículo complementario I. Instituto de Ecología A.C. Centro Regional del Bajío, Pátzcuaro.

Sánchez-Sánchez C.D. 2012. Diversidad florística y etnobotánica de los cultivos de Olea europea L. en El Olivo, Ixmiquilpan, Hidalgo. Tesis licenciatura. Facultad de Estudios Superiores Zaragoza, Universidad Nacional Autónoma de México, México, D.F. 131 pp.

Shreve F. y Wiggins I.L. 1964. Vegetation and Flora of the Sonoran Desert. Vol. II. Stanford University Press, Stanford.

Soriano-Martínez A.M y López-Soto M. M. 1994. Flora y relaciones fitogeográficas del Valle de Actopan, Hidalgo. Tesis licenciatura, Facultad de Estudios Superiores Zaragoza, Universidad Nacional Autónoma de México, México, D.F. 94 pp.

Sousa S.M. y Delgado S.A. 1993. Mexican Leguminosae: phytogeography, endemism, and origins. En: Ramamoorthy T.P., Bye R., Lot A. y Fa J. Eds. Biological Diversity of Mexico: Origins and Distribution, pp. 459-511, Oxford University Press, Nueva York.

Toledo V.M. y Ordoñez M.J. 1993. The biodiversity scenario of México: a review of terrestrial habitats. En: Ramamoorthy T.P., Bye R., Lot A. y Fa. J. Eds. Biological Diversity of Mexico: Origins and Distribution, pp. 757-777, Oxford University Press, Nueva York. 
Tropicos. Missouri Botanical Garden. <http://www.tropicos.org> (consultado septiembre 2012).

Valiente-Banuet A., Flores-Hernández N., Verdú M. y Dávila P. 1998. The chaparral vegetation in Mexico under nonmediterranean climate: The convergence and Madrean-Tethyan hypotheses reconsidered. American Journal of Botany 85:1398-1408.

Valiente-Banuet A., Casas A., Alcántara A., Dávila P., Flores-Hernández N., Arizmendi M.C., Villaseñor J.L. y Ortega-Ramírez J. 2000. La vegetación de Tehuacan-Cuicatlán. Boletín de la Sociedad Botánica de México 67:24-74.

Valiente-Banuet A., Solís L., Dávila P., Arizmendi M.C., Silva P.C., Ortega-Ramírez J., Treviño C.J., Rangel-Landa S. y Casas A. 2009. Guía de la Vegetación del Valle de Tehuacán-Cuicatlán. CONACYT-SEMARNAT, Fundación para la Reserva de la Biosfera Cuicatlán, A.C., Instituto de Ecología, Centro de Investigaciones en Ecosistemas, FES Iztacala, UNAM, CONABIO, México, D.F.

Velasco-Santiago C. y Ojeda-Rivera F. 1989. Clasificación y caracterización fisonómica de la vegetación del Valle del Mezquital, Hgo. Tesis de licenciatura, Escuela Nacional de Estudios Profesionales Iztacala, Universidad Nacional Autónoma de México. México, D.F. 160 pp.

Recibido: 25 de junio de 2012

Aceptado: 12 de diciembre de 2012
Villaseñor J.L. 2001. Catálogo de Autores de Plantas Vasculares de México. Instituto de Biología, Universidad Nacional Autónoma de México, México, D.F.

Villaseñor J.L. 2003. Diversidad y distribución de las Magnoliophyta de México. Interciencia 28:160-167.

Villaseñor J.L., Dávila P. y Chiang F. 1990. Fitogeografía del Valle de Tehuacan-Cuicatlán. Boletín de la Sociedad Botánica de México 50:135-149.

Villavicencio M.A., Pérez B.E. y Ramírez A.A. 1998. Lista Florística del Estado de Hidalgo. Centro de Investigaciones Biológicas, Universidad Autónoma del Estado de Hidalgo, Pachuca.

Whittaker R.H. 1975. Communities and Ecosystems. MacMillan Publishing Company, Inc., Nueva York.

Zamudio-Ruiz S. 1984. La vegetación de la cuenca del río Estórax en el estado de Querétaro y sus relaciones fitogeográficas. Tesis licenciatura, Facultad de Ciencias, Universidad Nacional Autónoma de México. México, D.F. 275 pp.

Zamudio S., Rzedowski J., Carranza E. y Calderón de Rzedowski G. 1992. La Vegetación en el Estado de Querétaro. Consejo de Ciencia y Tecnología del estado de Querétaro. Querétaro, Instituto de Ecología, A.C. Centro Regional Bajío, Pátzcuaro. 
Apéndice. Plantas vasculares del Valle de Tecozautla, Hidalgo. Colectores: Sonia Rojas (SR), Carlos Castillejos Cruz (CCC). Los ejemplares de respaldo están depositados en FEZA. Distribución geográfica (DG): 1 México, 1a norte al sur de México, 1b norte al centro de México, 1c centro de México, 1 d centro al sur de México; 2 Megaméxico 1, 2a sur de Estados Unidos de América a México, 2b sur de Estados Unidos de América al centro de México; 3 Megaméxico 2, 3a México al norte de Nicaragua, 3b centro de México al norte de Nicaragua; 4 Megaméxico 3; 5 Distribución continental; 6 Amplia distribución. Forma de crecimiento (FC): $\mathrm{A}=$ árbol, $\mathrm{Ar}=$ arbusto, $\mathrm{E}=$ epífita, $\mathrm{H}=$ hierba (anual y perenne), $\mathrm{P}=$ parásita, $\mathrm{At}=$ arrosetada, $\mathrm{S}=$ suculenta, $\mathrm{T}=$ trepadora. Tipo de vegetación (TV) donde se han registrado las especies en otros estudios: $\mathrm{BC}=$ bosque de coníferas, $\mathrm{BE}=$ bosque espinoso, BMM = bosque mesófilo de montaña, BQ = bosque de Quercus, BTC = bosque tropical caducifolio, BTP = bosque tropical perennifolio, $\mathrm{BTS}=$ bosque tropical subcaducifolio, $\mathrm{MX}=$ matorral xerófilo, $\mathrm{P}=$ pastizal, $\mathrm{VA}=$ vegetación acuática y subacuática. (*) Especies excluidas del análisis fitogeográfico.

\begin{tabular}{cccc}
\hline TAXA & FC & TV \\
\hline
\end{tabular}

\section{LICOPHYTA}

\section{SELAGINELLACEAE}

Selaginella lepidophylla (Hook. \& Grev.) Spring (SR 279)

$B E, B Q, B T C, M X$

\section{MONILOPHYTA}

\section{DRYOPTERIDACEAE}

Dryopteris wallichiana (Spreng.) Hyl. (SR 539)

BC, BTC, MX

\section{EQUISETACEAE}

Equisetum hyemale L. var. affine (Engelm.) A.A.Eaton (SR 280)

\section{MARSILEACEAE}

Marsilea mollis B.L.Rob. \& Fernald (SR 331)

\section{POLYPODIACEAE}

Polypodium madrense J.Sm. (SR 378)

P. polypodioides (L.) Watt (SR 237)

$\mathrm{H}$

$\mathrm{H}$

\section{PTERIDACEAE}

Bommeria pedata (Sw.) E.Fourn. (SR 141)

B. subpaleacea Maxon (SR 515)

Cheilanthes affinis Mett. (SR 186)

C. alabamensis (Buckley) Kunze (SR 527)

C. allosuroides Mett. (SR 276)

C. beitelii Mickel (SR 511)

C. bonariensis (Willd.) Proctor (SR 403)

C. crassifolia (Houlston \& T.Moore) Mickel \& Beitel (SR 215)

C. formosa (Liebm.) Mickel \& Beitel (SR 494)

C. incana (C. Presl) Mickel \& Beitel (SR 523)

C. lendigera (Cav.) Sw. (SR 308)

C. myriophylla Desv. (SR 300)

C. notholaenoides (Desv.) Maxon ex Weath. (SR 473)

C. pringlei Davenp. (SR 442)

C. sinuata (Lag. ex Sw.) Domin (SR 337)

C. sulphurea (Cav.) Mickel \& Beitel (SR 472)

\section{SALVINIACEAE}

Azolla microphylla Kaulf. (SR 118)

Salvinia minima Baker (SR 396)

\section{THELYPTERIDACEAE}

Thelypteris patens (Sw.) Small var. patens (SR 483)

\section{WOODSIACEAE}

Woodsia mollis (Kaulf.) J.Sm. (SR 482)
$\mathrm{H}$

$\mathrm{H}$

$\mathrm{H}$

$\mathrm{H}$

$\mathrm{H}$

$\mathrm{H}$

$\mathrm{H}$

$\mathrm{H}$

$\mathrm{H}$

$\mathrm{H}$

$\mathrm{H}$

$\mathrm{H}$

$\mathrm{H}$

$\mathrm{H}$

$\mathrm{H}$

$\mathrm{H}$

$\mathrm{H}$

$\mathrm{H}$

$\mathrm{H}$

$\mathrm{H}$

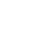

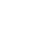

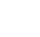

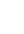

(1)

H

VA

MX

MX

BC, BTC, MX

$M X$

$M X$

$M X$

$M X, P$

$M X$

BTC, MX

$\mathrm{BC}, \mathrm{BQ}, \mathrm{BTC}, \mathrm{MX}$

MX

BTC, MX

$B C, B Q, B T C, M X$

$M X$

$M X$

MX

BTC, $M X$

$\mathrm{MX}$

VA

VA

MX

$M X, P$ 
Apéndice. Continuación

\section{TAXA}

FC

TV

\section{GIMNOSPERMAS \\ CUPRESSACEAE}

Juniperus flaccida Schltdl. var. flaccida (SR 492)

Taxodium mucronatum Ten. (SR 247)

A

A

\section{ANGIOSPERMAS MONOCOTILEDÓNEAS AMARYLLIDACEAE}

Allium glandulosum Link \& Otto (SR 167)

Manfreda guttata (Jacobi \& C.D.Bouché) Rose (CCC 1384, 1390)

Zephyranthes fosteri Traub (SR 6)

\section{ARACEAE}

Lemna gibba L. (SR 445)

\section{ASPARAGACEAE}

Agave applanata Lem. ex Jacobi (SR 556)

A. lechuguilla Torr. (SR 130)

A. macroculmis Tod. (SR 164)

A. striata Zucc. (SR 180)

Dasylirion acrotrichum (Schiede) Zucc. (SR 91)

Echeandia durangensis (Greenm.) Cruden (SR 163)

E. mexicana Cruden (SR 228)

E. nana (Baker) Cruden (SR 92)

Milla biflora Cav. (SR 171)

Yucca filifera Chabaud (SR 1)

\section{BROMELIACEAE}

Hechtia podantha Mez (SR 565)

Tillandsia recurvata (L.) L. (SR 2)

T. utriculata L. (SR 65)

\section{COMMELINACEAE}

Commelina diffusa Burm. f. (SR 223)

C. erecta L. (SR 173)

Gibasis linearis (Benth.) Rohweder (SR 392)

G. pellucida (M.Martens \& Galeotti) D.R.Hunt (SR 457)

G. pulchella (Kunth) Raf. (SR 236B)

Tinantia erecta (Jacq.) Schltdl. (SR 446)

Tradescantia crassifolia Cav. var. crassifolia (SR 218B)

T. velutina Kunth \& Bouché (SR 218)

Tripogandra purpurascens (S. Schauer) Handlos (SR 294)

\section{CYPERACEAE}

Cyperus hermaphroditus (Jacq.) Standl. (SR 250)

C. seslerioides Kunth (SR 230)

Eleocharis elegans (Kunth) Roem. \& Schult. (SR 780)

\section{IRIDACEAE}

Nemastylis tenuis (Herb.) S.Watson (SR 1008)

Sisyrinchium tenuifolium Humb. \& Bonpl. ex Willd. (SR 907)

\section{POACEAE}

Aegopogon cenchroides Humb. \& Bonpl. ex Willd. (SR 319)
$\mathrm{H}$

$\mathrm{H}$

$\mathrm{H}$

$\mathrm{H}$

At

At

At

At

$\mathrm{Ar}$

$\mathrm{H}$

$\mathrm{H}$

$\mathrm{H}$

$\mathrm{H}$

A

At

$\mathrm{H}$

$\mathrm{H}$

H

$\mathrm{H}$

$\mathrm{H}$

$\mathrm{H}$

$\mathrm{H}$

$\mathrm{H}$

$\mathrm{H}$

$\mathrm{H}$

$\mathrm{H}$

$\mathrm{H}$

$\mathrm{H}$

$\mathrm{H}$

$\mathrm{H}$

$\mathrm{H}$

$\mathrm{H}$
$B C, B Q, P$

$M X$

$M X, P$

$M X, P$

$B C, B Q, M X, P$

VA

MX

MX

$M X$

$B C, B M M, M X$

$B Q, M X$

$B C, B Q$

$\mathrm{BC}, \mathrm{BQ}$

$B C, B Q, P$

$B Q, M X, P$

$M X$

MX

$B C, B Q, M X$

$B C, B Q, M X$

MX, P

$M X$

$M X, P$

$B C, B Q, M X, P$

$$
P
$$

$M X$

$M X, P$

$M X, P$

$M X, P$

$\mathrm{BC}, \mathrm{BQ}, \mathrm{BMM}, \mathrm{MX}, \mathrm{P}$

$B C, B Q, M X, P$

$B C, B E, B Q, B M M, B T C, M X, P, V A$

$\mathrm{BQ}, \mathrm{MX}, \mathrm{P}$

$B C, B Q, M X, P$

$B Q, B T C, M X$ 
Apéndice. Continuación

\begin{tabular}{|c|c|c|}
\hline TAXA & FC & TV \\
\hline A. tenellus (DC.) Trin. (SR 342) & $\mathrm{H}$ & $\mathrm{BQ}, \mathrm{BTC}, \mathrm{P}$ \\
\hline Aristida laxa Cav. (SR 268) & $\mathrm{H}$ & $M X, P$ \\
\hline Arundo donax L. * (SR 467) & $\mathrm{H}$ & \\
\hline Bouteloua curtipendula (Michx.) Torr. (SR 338) & $\mathrm{H}$ & $M X, P$ \\
\hline B. hirsuta Lag. (SR 344) & $\mathrm{H}$ & $\mathrm{BC}, \mathrm{BTC}, \mathrm{MX}, \mathrm{P}$ \\
\hline B. repens (Kunth) Scribn. \& Merr. (SR 520) & $\mathrm{H}$ & $\mathrm{BC}, \mathrm{BE}, \mathrm{BQ}, \mathrm{BTC}, \mathrm{MX}, \mathrm{P}$ \\
\hline B. simplex Lag. (SR 133) & $\mathrm{H}$ & $\mathrm{BC}, \mathrm{MX}, \mathrm{P}$ \\
\hline Briza subaristata Lam. (SR 198) & $\mathrm{H}$ & $B Q, P$ \\
\hline Bromus anomalus Rupr. ex E.Fourn. (SR 263) & $\mathrm{H}$ & $\mathrm{BQ}, \mathrm{P}$ \\
\hline Buchloe dactyloides (Nutt.) Engelm. (SR 354) & $\mathrm{H}$ & $\mathrm{P}$ \\
\hline Cenchrus ciliaris L. * (SR 203) & $\mathrm{H}$ & \\
\hline Chloris submutica Kunth (SR 143) & $\mathrm{H}$ & $B Q, M X, P$ \\
\hline C. virgata Sw. (SR 340) & $\mathrm{H}$ & $M X, P$ \\
\hline Cynodon dactylon (L.) Pers. (SR 117) & $\mathrm{H}$ & $M X, P$ \\
\hline Digitaria argillacea (Hitchc. \& Chase) Fernald (SR 97) & $\mathrm{H}$ & $\mathrm{BQ}, \mathrm{BTC}, \mathrm{P}$ \\
\hline D. horizontalis Willd. (SR 533) & $\mathrm{H}$ & MX \\
\hline Enneapogon desvauxii P.Beauv. (SR 303) & $\mathrm{H}$ & $M X, P$ \\
\hline Eragrostis curvula (Schrad.) Nees (SR 525) & $\mathrm{H}$ & $\mathrm{MX}, \mathrm{P}$ \\
\hline E. intermedia Hitchc. var. intermedia (SR 428) & $\mathrm{H}$ & $B C, B Q, M X, P$ \\
\hline E. tenuifolia (A.Rich.) Hochst. ex Steud. (SR 410) & $\mathrm{H}$ & MX \\
\hline Hilaria cenchroides Kunth (SR 282) & $\mathrm{H}$ & $\mathrm{BC}, \mathrm{BQ}, \mathrm{MX}, \mathrm{P}$ \\
\hline Leptochloa fusca (L.) Kunth subsp. uninervia (J.Presl) N.W.Snow (SR 262) & $\mathrm{H}$ & $\mathrm{BC}, \mathrm{BE}, \mathrm{BQ}$ \\
\hline Lycurus phalaroides Kunth (SR 190) & $\mathrm{H}$ & $B C, B Q, M X, P$ \\
\hline Melinis repens (Willd.) Zizka * (SR 39) & $\mathrm{H}$ & \\
\hline Microchloa kunthii Desv. (SR 355) & $\mathrm{H}$ & $B C, B Q, M X, P$ \\
\hline Muhlenbergia articulata Scribn. (SR 504) & $\mathrm{H}$ & $\mathrm{BQ}$ \\
\hline M. dubia E. Fourn. (SR 374) & $\mathrm{H}$ & $\mathrm{BQ}, \mathrm{P}$ \\
\hline M. emersleyi Vasey (SR 255) & $\mathrm{H}$ & $B C, B Q, M X, P$ \\
\hline M. pusilla Steud. (SR 495) & $\mathrm{H}$ & $\mathrm{BQ}, \mathrm{BTC}, \mathrm{MX}, \mathrm{P}$ \\
\hline M. rigida (Kunth) Kunth (SR 486) & $\mathrm{H}$ & $\mathrm{BQ}, \mathrm{BTC}, \mathrm{MX}, \mathrm{P}$ \\
\hline M. spiciformis Trin. (SR 329) & $\mathrm{H}$ & $\mathrm{BQ}$ \\
\hline M. tenuifolia (Kunth) Kunth (SR 461) & $\mathrm{H}$ & $M X, P$ \\
\hline Nassella mucronata (Kunth) R.W.Pohl (SR 304) & $\mathrm{H}$ & $B C, B Q, M X, P$ \\
\hline Panicum obtusum Kunth (SR 161) & $\mathrm{H}$ & $B C, B Q, M X, P$ \\
\hline Paspalum adoperiens (E.Fourn.) Chase (SR 197) & $\mathrm{H}$ & MX \\
\hline P. notatum A.H.Liogier ex Flüggé (SR 144) & $\mathrm{H}$ & $B C, B Q, M X, P$ \\
\hline Pennisetum villosum R.Br. ex Fresen. (SR 517) & $\mathrm{H}$ & $M X, P$ \\
\hline Poa annua L. (SR 464) & $\mathrm{H}$ & $\mathrm{BC}, \mathrm{BMM}, \mathrm{BQ}, \mathrm{MX}, \mathrm{P}$ \\
\hline Schizachyrium condensatum (Kunth) Nees (SR 425) & $\mathrm{H}$ & $\mathrm{BC}$ \\
\hline Setaria grisebachii E.Fourn. (SR 352) & $\mathrm{H}$ & MX \\
\hline S. macrostachya Kunth (SR 341) & $\mathrm{H}$ & $M X, P$ \\
\hline S. parviflora (Poir.) Kerguélen (SR 360) & $\mathrm{H}$ & $M X, P$ \\
\hline Setariopsis latiglumis (Vasey) Scribn. (SR 135B) & $\mathrm{H}$ & $M X$ \\
\hline Sorghastrum brunneum Swallen (SR 161) & $\mathrm{H}$ & $\mathrm{BC}$ \\
\hline Vulpia myuros (L.) C.C.Gmel. (SR 514) & $\mathrm{H}$ & $\mathrm{BC}, \mathrm{BQ}$ \\
\hline \multicolumn{3}{|l|}{ EUDICOTILEDÓNEAS } \\
\hline \multicolumn{3}{|l|}{ ACANTHACEAE } \\
\hline Anisacanthus quadrifidus (Vahl) Ness var. quadrifidus (SR 421) & Ar & $\mathrm{BE}, \mathrm{BQ}, \mathrm{MX}, \mathrm{P}$ \\
\hline Carlowrightia neesiana (Schauer ex Nees) T.F.Daniel (SR 437) & $\mathrm{H}$ & $\mathrm{BE}, \mathrm{BTC}, \mathrm{MX}, \mathrm{P}$ \\
\hline
\end{tabular}


Apéndice. Continuación

\begin{tabular}{|c|c|c|}
\hline TAXA & FC & TV \\
\hline C. lindauiana Standl. (SR 265) & $\mathrm{H}$ & $B E, M X, P$ \\
\hline Dicliptera peduncularis Nees (SR 236) & $\mathrm{H}$ & $M X, P$ \\
\hline Dyschoriste microphylla Kuntze (SR 179) & $\mathrm{H}$ & $B Q, M X$ \\
\hline Hoverdenia speciosa Nees (SR 547) & $\mathrm{Ar}$ & BTC, MX \\
\hline Justicia caudata A.Gray (SR 330) & $\mathrm{H}$ & MX \\
\hline Ruellia lactea Cav. (SR 79) & $\mathrm{H}$ & $M X, P$ \\
\hline R. speciosa Mart. ex Ness (SR 291) & $\mathrm{Ar}$ & $M X, P$ \\
\hline Tetramerium nervosum Nees (SR 278) & $\mathrm{H}$ & $M X, P$ \\
\hline Thunbergia alata Bojer ex Sims * (SR 450) & $\mathrm{T}$ & \\
\hline \multicolumn{3}{|l|}{ AMARANTHACEAE } \\
\hline Amaranthus palmeri S.Watson (SR 364) & $\mathrm{H}$ & $M X, P$ \\
\hline Chenopodium fremontii S.Watson (SR 433) & $\mathrm{H}$ & MX \\
\hline C. graveolens Willd. (SR 524) & $\mathrm{H}$ & $M X$ \\
\hline Gomphrena decumbens Jacq. (SR 206) & $\mathrm{H}$ & $M X, P$ \\
\hline Iresine cassiniiformis S.Schauer (SR 471) & $\operatorname{Ar}$ & MX \\
\hline I. diffusa Humb. \& Bonpl. ex Willd. (SR 419) & $\mathrm{H}$ & $M X$ \\
\hline I. heterophylla Standl. (SR 424) & $\mathrm{H}$ & $M X$ \\
\hline I. schaffneri S.Watson (SR 264) & $\mathrm{H}$ & $M X$ \\
\hline Kochia scoparia (L.) Schrad. * (SR 16) & $\operatorname{Ar}$ & \\
\hline Salsola tragus L. ${ }^{*}($ SR 201$)$ & $\operatorname{Ar}$ & \\
\hline \multicolumn{3}{|l|}{ ANACAMPSEROTACEAE } \\
\hline Talinopsis frutescens A.Gray (SR 578) & $\mathrm{Ar}$ & $M X$ \\
\hline \multicolumn{3}{|l|}{ ANACARDIACEAE } \\
\hline Pistacia mexicana Kunth (SR 440) & A & BTC, MX \\
\hline Schinus molle L. * (SR 369) & A & \\
\hline Toxicodendron radicans (L.) Kuntze (SR 551) & A & BMM \\
\hline \multicolumn{3}{|l|}{ APOCYNACEAE } \\
\hline Asclepias angustifolia Schweigg. (SR 540) & $\mathrm{H}$ & MX \\
\hline A. curassavica L. (SR 222) & $\mathrm{H}$ & $M X, P$ \\
\hline A. linaria Cav. (SR 9) & $\mathrm{H}$ & $M X, P$ \\
\hline A. oenotheroides Schltdl. \& Cham. (SR 127) & Ar & MX \\
\hline Matelea schaffneri (A.Gray ex Hemsl.) Woodson (SR 200) & $\mathrm{T}$ & $M X$ \\
\hline Metastelma mexicanum (Brandegee) Fishbein \& R.A.Levin (SR 309) & $\mathrm{T}$ & $\mathrm{BC}, \mathrm{BE}, \mathrm{BMM}, \mathrm{BQ}, \mathrm{BTC}, \mathrm{MX}, \mathrm{P}$ \\
\hline Stapelia gigantea N.E. Br. * (SR 108) & $\mathrm{Ar}$ & \\
\hline Telosiphonia lanuginosa (M.Martens \& Galeotti) Henr. (SR 238) & $\mathrm{Ar}$ & $M X$ \\
\hline \multicolumn{3}{|l|}{ ASTERACEAE } \\
\hline Adenophyllum cancellatum (Cass.) Villarreal (SR 359) & $\mathrm{H}$ & $M X, P$ \\
\hline A. porophyllum (Cav.) Hemsl. (SR 249) & $\mathrm{H}$ & $M X, P$ \\
\hline $\begin{array}{l}\text { Ageratina espinosarum (A.Gray) R.M.King \& H.Rob. } \\
\text { var. espinosarum (SR 544) }\end{array}$ & $\mathrm{Ar}$ & $B Q, M X$ \\
\hline A. hidalgensis (H.Rob.) R.M.King \& H.Rob. (SR 274) & $\mathrm{Ar}$ & $\mathrm{BC}, \mathrm{BQ}$ \\
\hline Ageratum corymbosum Zuccagni (SR 388) & $\mathrm{H}$ & $M X, P$ \\
\hline Aldama dentata La Llave (SR 541) & $\mathrm{H}$ & $M X, V A$ \\
\hline Aster subulatus Michx. var. subulatus (SR 193) & $\operatorname{Ar}$ & MX \\
\hline Baccharis pteronioides DC. (SR 85) & $\operatorname{Ar}$ & MX \\
\hline Bahia pringlei Greenm. (SR 543) & $\operatorname{Ar}$ & MX \\
\hline Barkleyanthus salicifolius (Kunth) H.Rob. \& Brettell (SR 339) & $\operatorname{Ar}$ & $B C, B Q, M X, P$ \\
\hline
\end{tabular}


Apéndice. Continuación

\begin{tabular}{|c|c|c|}
\hline TAXA & FC & TV \\
\hline Bidens odorata Cav. var. odorata (SR 322) & $\mathrm{H}$ & MX \\
\hline Calyptocarpus vialis Less. * (SR 187) & $\mathrm{H}$ & \\
\hline Chaetopappa ericoides (Torr.) G.L.Nesom (SR 195) & $\mathrm{H}$ & $M X, P$ \\
\hline Chromolaena collina (DC.) R.M.King \& H.Rob. (SR 335) & Ar & $\mathrm{BC}, \mathrm{BQ}, \mathrm{BTC}, \mathrm{BTS}, \mathrm{P}$ \\
\hline Chrysactinia mexicana A.Gray (SR 479) & $\mathrm{Ar}$ & $\mathrm{BC}, \mathrm{BQ}, \mathrm{MX}, \mathrm{P}$ \\
\hline Coreopsis mutica DC. var. mutica (SR 336) & $\operatorname{Ar}$ & $B Q, M X, P$ \\
\hline Dahlia sorensenii H.V.Hansen \& Hjert. (SR 115) & $\mathrm{H}$ & $\mathrm{BE}, \mathrm{MX}, \mathrm{P}$ \\
\hline Dyssodia papposa (Vent.) Hitchc. * (SR 420) & $\mathrm{Ar}$ & \\
\hline D. pinnata (Cav.) B.L.Rob. var. pinnata (SR 418) & Ar & $\mathrm{BC}, \mathrm{BQ}, \mathrm{MX}, \mathrm{P}$ \\
\hline Erigeron pubescens Kunth (SR 66) & $\mathrm{H}$ & $\mathrm{BC}, \mathrm{BE}, \mathrm{BQ}, \mathrm{MX}, \mathrm{P}$ \\
\hline Eutetras pringlei Greenm. (SR 500) & $\operatorname{Ar}$ & $M X, P$ \\
\hline Fleischmannia pycnocephala (Less.) R.M.King \& H.Rob. (SR 439) & $\mathrm{H}$ & $B C, B Q, M X, P$ \\
\hline Florestina pedata (Cav.) Cass. (SR 254) & $\operatorname{Ar}$ & $M X, P$ \\
\hline Helianthus annuus L. (SR 379) & $\mathrm{H}$ & $M X, P$ \\
\hline Heterosperma pinnatum Cav. (SR 257) & $\mathrm{H}$ & $B C, B Q, M X, P$ \\
\hline Heterotheca inuloides Cass. (SR 491) & $\mathrm{H}$ & MX \\
\hline Lasianthaea aurea (D.Don) K.M.Becker (SR 99) & $\mathrm{H}$ & $M X, P$ \\
\hline Melampodium divaricatum (Rich.) DC. (SR 460B) & $\mathrm{H}$ & $M X, P$ \\
\hline M. sericeum Lag. (SR 192) & $\mathrm{H}$ & MX \\
\hline Montanoa tomentosa Cerv. subsp. tomentosa (SR 283) & $\mathrm{Ar}$ & MX \\
\hline Parthenium bipinnatifidum (Ortega) Rollins (SR 11) & $\mathrm{H}$ & $M X$ \\
\hline P. incanum Kunth (SR 158) & $\operatorname{Ar}$ & $M X, P$ \\
\hline Perymenium mendezii DC. var. mendezii (SR 347) & Ar & MX \\
\hline Piqueria trinervia Cav. (SR 490) & $\mathrm{H}$ & $B C, B Q, M X, P$ \\
\hline Porophyllum linaria (Cav.) DC. (SR 384) & $\mathrm{H}$ & $\mathrm{P}$ \\
\hline Sanvitalia procumbens Lam. (SR 101) & $\mathrm{H}$ & $M X, P$ \\
\hline $\begin{array}{l}\text { Schkuhria pinnata (Lam.) Kuntze ex Thell. } \\
\text { var. wislizeni (A. Gray) B.L.Turner (SR 534) }\end{array}$ & $\mathrm{H}$ & $M X, P$ \\
\hline Spilanthes oppositifolia (Lam.) D'Arcy (SR 373) & A & VA \\
\hline Stevia incognita Grashoff (SR 513) & $\mathrm{H}$ & $\mathrm{BC}$ \\
\hline Tagetes erecta L. (SR 468) & $\mathrm{H}$ & $M X, P$ \\
\hline T. lunulata Ortega (SR 260) & $\mathrm{H}$ & $B E, B Q, M X, P$ \\
\hline Thymophylla acerosa (DC.) Strother (SR 571) & $\operatorname{Ar}$ & $\mathrm{MX}, \mathrm{P}$ \\
\hline T. pentachaeta (DC.) Small var. pentachaeta (SR 502) & $\mathrm{H}$ & MX \\
\hline T. setifolia Lag. (SR 266) & Ar & $M X, P$ \\
\hline Tithonia tubiformis (Jacq.) Cass. (SR 288) & $\mathrm{H}$ & $\mathrm{BC}, \mathrm{BE}, \mathrm{BQ}, \mathrm{BTC}, \mathrm{MX}, \mathrm{P}$ \\
\hline Townsendia mexicana A.Gray (SR 437) & $\operatorname{Ar}$ & $M X$ \\
\hline Tridax coronopifolia (Kunth) Hemsl. (SR 126) & Ar & $M X, P$ \\
\hline T. rosea Sch.Bip. ex B.L.Rob. \& Greenm. (SR 522) & $\mathrm{H}$ & MX \\
\hline Trixis inula Crantz & $\operatorname{Ar}$ & $M X$ \\
\hline Verbesina encelioides (Cav.) Benth. \& Hook. f. ex A.Gray (SR 460) & Ar & $M X, P$ \\
\hline V. hypomalaca B.L.Rob. \& Greenm. (SR 285) & $\mathrm{H}$ & $\mathrm{BE}, \mathrm{BQ}, \mathrm{MX}$ \\
\hline V. oncophora B.L.Rob. \& Seaton (SR 372) & $\mathrm{Ar}$ & $\mathrm{BC}, \mathrm{BQ}, \mathrm{BMM}$ \\
\hline V. serrata Cav. (SR 381) & Ar & MX \\
\hline V. virgata Cav. var. virgata (SR 380) & $\operatorname{Ar}$ & $B Q, M X, P$ \\
\hline Viguiera buddlejiformis (DC.) Benth. \& Hook. f. ex Hemsl. (SR 240) & Ar & $M X, P$ \\
\hline V. cordata (Hook. \& Arn.) D’Arcy var. cordata (SR 261) & $\mathrm{H}$ & $B Q, P$ \\
\hline V. dentata (Cav.) Spreng. (SR 299) & $\mathrm{H}$ & MX \\
\hline Wedelia acapulcensis Kunth var. hispida (Kunth) Strother (SR 122) & Ar & $M X, P$ \\
\hline Xanthium strumarium L. (SR 301) & Ar & $M X, P$ \\
\hline
\end{tabular}


Apéndice. Continuación

TAXA FC

Zaluzania augusta (Lag.) Sch.Bip. var. augusta (SR 394)

Z. triloba (Ortega) Pers. (SR 526)

Zinnia peruviana L. (SR 98)

Tecoma stans (L.) Juss. ex Kunth var. stans (SR 194)

\section{BORAGINACEAE}

Heliotropium foliosissimum J.F.Macbr. (SR 426)

H. pringlei B.L.Rob. (SR 119)

Nama dichotoma (Ruiz \& Pav.) Choisy var. dichotoma (SR 562)

N. origanifolia Kunth (SR 112)

N. palmeri A.Gray ex Hemsl. (SR 427)

N. undulata Kunth (SR 146)

\section{BRASSICACEAE}

Lepidium schaffneri Thell. (SR 503)

L. virginicum L. var. virginicum (SR 459)

Lesquerella fendleri (A.Gray) S.Watson (SR 156)

Raphanus raphanistrum L. * (SR 136)

Sisymbrium irio L. * (SR 536)

\section{BURSERACEAE}

Bursera cuneata (Schltdl.) Engl. (SR 582)

B. fagaroides (Kunth) Engl. var. fagaroides (SR 30)

\section{CACTACEAE}

Coryphantha radians (DC.) Britton \& Rose (SR 32)

C. ottonis (Pfeiff.) Lem. (SR 202)

Cylindropuntia imbricata (Haw.) F.M.Knuth subsp. imbricata (SR 28B)

Echinocactus grusonii Hildm. (SR 35)

E. platyacanthus Link \& Otto (SR 33)

Echinocereus cinerascens Lem. var. cinerascens (SR 27)

Ferocactus histrix (DC.) G.E.Linds. (SR 61B)

F. latispinus (Haw.) Britton \& Rose (SR 40)

Mammillaria compressa DC. subsp. compressa (SR 563)

M. decipiens Scheidw. subsp. camptotricha (Dams) D.R.Hunt (SR 555)

M. elongata DC. subsp. elongata (SR 90)

M. longimamma DC. var. longimamma (SR 5)

M. polythele Mart. subsp. polythele (SR 229)

M. zephyranthoides Scheidw. (SR 61)

Marginatocereus marginatus (DC.) Backeb. (SR 443)

Myrtillocactus geometrizans (Mart. ex Pfeiff.) Console (SR 41)

Neolloydia conoidea (DC.) Britton \& Rose (SR 118B)

Opuntia durangensis Britton \& Rose (SR 89B)

O. imbricata (Haw.) DC. (SR 28)

O. joconostle F.A.C.Weber (SR 26)

O. microdasys (Lehm.) Pfeiff. var. minor Salm-Dyck (SR 93)

O. robusta J.C.Wendl. (SR 71)

O. tomentosa Salm-Dyck (SR 89)

Pachycereus weberi (J.M.Coult.) Backeb. (SR 134)

Stenocactus pentacanthus (Lem.) A.Berger ex A.W.Hill (SR 52)

\section{FC}

Ar

Ar

$\mathrm{H}$

$\operatorname{Ar}$

TV

$B C, B Q, M X$
$M X$
$M X, P$

$M X, P$

$\mathrm{H}$

$\mathrm{H}$

$\mathrm{P}$

$M X, P$

$M X, P$

$B Q, M X$

$M X$

MX

BQ, BTC, MX, P

$B C, M X, P$

$M X, P$

$\mathrm{H}$

$\mathrm{H}$

$\mathrm{H}$

A

Ar

$M X$

$M X, P$

S

S

$\mathrm{Ar}$

$S$

S

$S$

$S$

S

$S$

S

S

S

S

S

$\mathrm{Ar}$

A

S

$\mathrm{Ar}$

$\mathrm{Ar}$

A

$\mathrm{Ar}$

Ar

Ar

$\mathrm{Ar}$

S
$M X$
$M X, P$
$M X, P$
$M X$
MX
$M X, P$
$M X, P$
$M X, P$
MX
$M X, P$
$M X, P$
$M X$
MX, P
MX
MX
MX
MX
$M X, P$
$M X, P$
MX
BE
MX
$M X, P$
$M X$
$M X, P$ 
Apéndice. Continuación

\begin{tabular}{|c|c|c|}
\hline TAXA & FC & TV \\
\hline Stenocereus dumortieri (Scheidw.) Buxb. (SR 560) & A & MX \\
\hline $\begin{array}{l}\text { Thelocactus leucacanthus (Zucc. ex Pfeiff.) Britton \& Rose } \\
\text { subsp. schmollii (Werderm.) Mosco \& Zanov. (SR 23) }\end{array}$ & $\mathrm{S}$ & $M X, P$ \\
\hline \multicolumn{3}{|l|}{ CAMPANULACEAE } \\
\hline Lobelia xalapensis Kunth (SR 481) & $\mathrm{H}$ & BMM \\
\hline \multicolumn{3}{|l|}{ CANNABACEAE } \\
\hline Celtis reticulata Torr. (SR 581) & A & $\mathrm{BC}, \mathrm{BQ}, \mathrm{P}$ \\
\hline \multicolumn{3}{|l|}{ CARYOPHYLLACEAE } \\
\hline Drymaria effusa A.Gray (SR 43) & $\mathrm{H}$ & $\mathrm{BC}$ \\
\hline D. laxiflora Benth. (SR 548) & $\mathrm{H}$ & MX \\
\hline D. molluginea (Ser.) Didr. (SR 532) & $\mathrm{H}$ & MX \\
\hline D. tenuis S.Watson (SR 574) & $\mathrm{H}$ & MX \\
\hline \multicolumn{3}{|l|}{ CLEOMACEAE } \\
\hline Polanisia uniglandulosa (Cav.) DC. (SR 60) & $\mathrm{H}$ & MX \\
\hline \multicolumn{3}{|l|}{ CONVOLVULACEAE } \\
\hline Cuscuta obtusiflora Kunth (SR 325) & $\mathrm{P}$ & MX \\
\hline Evolvulus alsinoides (L.) L. (SR 142) & $\mathrm{H}$ & $\mathrm{BC}, \mathrm{MX}, \mathrm{P}$ \\
\hline Ipomoea capillacea (Kunth) G.Don (SR 29) & $\mathrm{H}$ & $M X, P$ \\
\hline I. cristulata Hallier f. (SR 395) & $\mathrm{T}$ & $M X, P$ \\
\hline I. murucoides Roem. \& Schult. (SR 362) & A & MX \\
\hline I. orizabensis (G.Pelletan) Ledeb. ex Steud. (SR 512) & $\mathrm{T}$ & $B C, B Q, M X, P$ \\
\hline I. plummerae A.Gray (SR 395) & $\mathrm{T}$ & $M X$ \\
\hline I. purpurea (L.) Roth (SR 289) & $\mathrm{T}$ & $B Q, M X, P$ \\
\hline \multicolumn{3}{|l|}{ CRASSULACEAE } \\
\hline Echeveria bifida Schltdl. (SR 498) & $\mathrm{H}$ & MX \\
\hline E. minima J. Meyrán subsp. minima (SR 445) & $\mathrm{H}$ & $M X$ \\
\hline E. paniculata A.Gray var. maculata Kimnach (SR 290) & $\mathrm{H}$ & $M X$ \\
\hline E. platyphylla Rose (SR 570) & $\mathrm{H}$ & MX \\
\hline E. secunda Booth ex Lindl. (SR 103) & $\mathrm{H}$ & $\mathrm{BC}, \mathrm{BQ}, \mathrm{MX}, \mathrm{P}$ \\
\hline Kalanchoe delagoensis Eckl. \& Zeyh. * (SR 583) & $\mathrm{H}$ & \\
\hline Pachyphytum glutinicaule Moran (CCC 3186) & $\mathrm{H}$ & MX \\
\hline Sedum bourgaei Hemsl. (SR 483) & $\operatorname{Ar}$ & $\mathrm{BC}, \mathrm{MX}$ \\
\hline S. corynephyllum Fröd. (SR 554) & $\mathrm{H}$ & MX \\
\hline $\begin{array}{l}\text { S. dendroideum Moc. \& Sessé ex DC. } \\
\text { subsp. parvifolium R.T.Clausen (SR 585) }\end{array}$ & $\mathrm{H}$ & $B C, M X$ \\
\hline S. ebracteatum Moc. \& Sessé ex. DC. subsp. ebracteatum (SR 431) & $\mathrm{H}$ & MX \\
\hline S. humifusum Rose (SR 233) & $\mathrm{H}$ & MX \\
\hline S. jurgensenii (Hemsl.) Moran subsp. jurgensenii (SR 389) & $\mathrm{H}$ & $M X$ \\
\hline $\begin{array}{l}\text { S. praealtum A.DC. subsp. parvifolium (R.T.Clausen) } \\
\text { R.T.Clausen (SR 105) }\end{array}$ & $\operatorname{Ar}$ & $\mathrm{BC}, \mathrm{BQ}, \mathrm{MX}$ \\
\hline Villadia misera (Lindl.) R.T.Clausen (SR 270) & $\mathrm{H}$ & MX \\
\hline \multicolumn{3}{|l|}{ CUCURBITACEAE } \\
\hline Cucurbita foetidissima Kunth * (SR 441) & $\mathrm{H}$ & \\
\hline Sicyos laciniatus L. (SR 224) & $\mathrm{H}$ & $M X, P$ \\
\hline \multicolumn{3}{|l|}{ EBENACEAE } \\
\hline Diospyros ebenaster Retz. * (SR 469) & A & \\
\hline
\end{tabular}


Apéndice. Continuación

TAXA FC

\section{EUPHORBIACEAE}

Acalypha brevicaulis Müll.Arg. (SR 133B)

A. mollis Kunth (SR 199)

A. monostachya Cav. (SR 149)

A. phleoides Cav. (SR 219)

A. subviscida S.Watson (SR 258)

Croton ciliatoglanduliferus Ortega (SR 54)

C. ehrenbergii Schltdl. (SR 11)

C. morifolius Willd. (SR 416)

Ditaxis heterantha Zucc. (CCC 3187)

Euphorbia antisiphylitica Zucc. (SR 170)

E. cyathophora Murray (SR 411)

E. dentata Michx. (SR 314)

E. hirta L. var. hirta (SR 407)

E. hirta L. var. procumbens (DC.) N.E.Br. (SR 407B)

E. nutans Lag. (SR 196)

E. serpens Kunth (SR 76)

Jatropha dioica Cerv. var. dioica (SR 4)

Ricinus communis L. * (SR 305)

Tragia nepetifolia Cav. (SR 24, 46, 83, 110)

\section{FABACEAE}

Acacia farnesiana (L.) Willd. (SR 87, 96, 360, 476, 580)

A. schaffneri (S.Watson) F.J.Herm. (SR 421)

A. tenuifolia (L.) Willd. (SR 184)

Acaciella angustissima (Mill.) Britton \& Rose (SR 239)

Brongniartia argentea Rydb. (SR 53)

B. foliolosa Benth. ex Hemsl. (SR 243, 400)

Calliandra humilis Benth. (SR 172, 242, 406)

Canavalia villosa Benth. (SR 63)

Centrosema pubescens Benth. (SR 399)

Crotalaria pumila Ortega (SR 465, 531)

Dalea bicolor Humb. \& Bonpl. ex Willd. (SR 385, 417)

D. melantha S.Schauer (SR 518)

D. prostrata Ortega (SR 487)

D. versicolor Zucc. var. involuta (Rydb.) Barneby (SR 175, 235)

Erythrina americana Mill. (SR 38, 80)

Eysenhardtia polystachya (Ortega) Sarg. (SR 107, 318)

E. punctata Pennell (SR 246)

Hoffmannseggia oxycarpa Benth.

subsp. arida (Rose) B.B.Simpson (SR 137, 160)

Indigofera suffruticosa Mill. (SR 244)

Lotus repens (G. Don) Sessé \& Moc. ex Standl. \& Steyerm. (SR 553)

Macroptilium gibbosifolium (Ortega) A.Delgado

(SR 277, 387, 398, 564, 576)

M. lathyroides (L.) Urb. (SR 402, 528)

Medicago sativa L. * (SR 556)

Mimosa biuncifera Benth. (SR 12, 177)

M. depauperata Benth. (SR 36, 121, 138)

Nissolia fruticosa Jacq. (SR 281)

\section{$\mathrm{H}$}

$\mathrm{Ar}$

$\mathrm{H}$

$\mathrm{H}$

Ar

Ar

$\mathrm{Ar}$

Ar

Ar

Ar

$\mathrm{H}$

$\mathrm{H}$

$\mathrm{H}$

$\mathrm{Ar}$

$\mathrm{H}$

$\mathrm{Ar}$

Ar

$\mathrm{H}$

Ar

$\mathrm{Ar}$

Ar

Ar

Ar

Ar

Ar

$\mathrm{H}$

$\mathrm{H}$

$\mathrm{H}$

Ar

$\mathrm{H}$

$\mathrm{H}$

$\mathrm{Ar}$

A

A

A

$\mathrm{H}$

Ar

T

$\mathrm{H}$

$\mathrm{H}$

$\mathrm{H}$

A

$\mathrm{Ar}$

T
TV

BE, MX

$B C, B Q$

$M X$

$B Q, M X, P$

$M X$

BE, BTC, BTS, MX

$M X, P$

$M X$

$M X, P$

$M X, P$

$B C, B Q, P$

$M X$

$\mathrm{P}$

$B Q, M X, P$

$M X, P$

$M X$

MX, P

$M X$

$M X, P$

BTC, MX

$M X, P$

$B Q, M X, P$

$B E, M X, P$

$M X, P$

BTC, MX, P

BC, BTC

BTC, MX, P

$M X, P$

$B C, B Q, B T C, M X, P$

$B C, B Q, M X, P$

$B C, B Q, M X, P$

$M X$

$M X, P$

$M X, P$

$M X$

BTC, MX, P

$B Q, B T C, B T S, M X$

$M X, P$

BTC, MX, P

MX

MX

BTC, P 
Apéndice. Continuación

\begin{tabular}{|c|c|c|}
\hline TAXA & FC & TV \\
\hline N. pringlei Rose (SR 508) & $\mathrm{T}$ & $M X, P$ \\
\hline Painteria elachistophylla (A.Gray ex S. Watson) Britton \& Rose (SR 178) & A & BTC, MX, P \\
\hline Pomaria melanosticta S.Schauer (SR 129, 176, 328) & $\operatorname{Ar}$ & MX \\
\hline $\begin{array}{l}\text { Prosopis laevigata (Humb. \& Bonpl. ex Willd.) } \\
\text { M.C.Johnst. (SR 430, 561) }\end{array}$ & A & $M X, P$ \\
\hline Rhynchosia prostrata Brandegee (SR 391) & $\mathrm{H}$ & $M X, P$ \\
\hline Senna septemtrionalis (Viv.) H.S.Irwin \& Barneby (SR 401) & Ar & $\mathrm{BC}, \mathrm{BMM}, \mathrm{BQ}, \mathrm{BTC}$ \\
\hline $\begin{array}{l}\text { S. wislizeni (A.Gray) H.S.Irwin \& Barneby var. painteri } \\
\text { (Britton) H.S.Irwin \& Barneby (SR 94) }\end{array}$ & A & MX \\
\hline \multicolumn{3}{|l|}{ FOUQUIERIACEAE } \\
\hline Fouquieria formosa Kunth (SR 25) & Ar & MX \\
\hline F. splendens Engelm. (SR 239) & $\mathrm{Ar}$ & MX \\
\hline \multicolumn{3}{|l|}{ GERANIACEAE } \\
\hline Erodium cicutarium (L.) L'Hér. ex Aiton * (SR 148) & $\mathrm{H}$ & \\
\hline \multicolumn{3}{|l|}{ KRAMERIACEAE } \\
\hline Krameria cytisoides Cav. (SR 226) & Ar & $\mathrm{BQ}, \mathrm{MX}$ \\
\hline K. grayi Rose \& Painter (SR 174) & $\operatorname{Ar}$ & MX \\
\hline \multicolumn{3}{|l|}{ LAMIACEAE } \\
\hline Hyptis albida Kunth (SR 292) & Ar & BTC, MX \\
\hline H. tomentosa Poit. (SR 573) & Ar & $M X, P$ \\
\hline Leonotis nepetifolia (L.) R.Br. * (SR 449) & $\mathrm{H}$ & \\
\hline Salvia melissodora Lag. (SR 68, 188, 272) & $\mathrm{H}$ & MX \\
\hline S. microphylla Kunth (SR 489) & $\mathrm{H}$ & $B Q, M X, P$ \\
\hline S. mocinoi Benth. (SR 154) & $\mathrm{H}$ & $\mathrm{BMM}, \mathrm{BQ}$ \\
\hline S. oreopola Fernald (SR 454) & $\mathrm{H}$ & $\mathrm{BC}$ \\
\hline S. reflexa Hornem. (SR 295) & $\mathrm{H}$ & $\mathrm{MX}$ \\
\hline S. tiliifolia Vahl (SR 293) & $\mathrm{H}$ & $\mathrm{BC}, \mathrm{BE}, \mathrm{BQ}, \mathrm{BTC}, \mathrm{MX}, \mathrm{P}$ \\
\hline Stachys coccinea Ortega (SR 125) & $\mathrm{H}$ & $B Q, M X, P$ \\
\hline \multicolumn{3}{|l|}{ LAURACEAE } \\
\hline Persea americana Mill. * (SR 550) & A & \\
\hline \multicolumn{3}{|l|}{ LINACEAE } \\
\hline Linum scabrellum Planch. (SR 435) & $\mathrm{H}$ & $B C, M X, P$ \\
\hline \multicolumn{3}{|l|}{ LOASACEAE } \\
\hline Cevallia sinuata Lag. (SR 204) & $\mathrm{H}$ & $M X, P$ \\
\hline Eucnide hirta (Pav. ex G. Don) H.J.Thomps. \& W.R.Ernst (SR 497) & $\mathrm{H}$ & MX \\
\hline E. lobata (Hook.) A.Gray (SR 45) & $\mathrm{H}$ & MX \\
\hline Mentzelia hispida Willd. $(245,297,326)$ & $\mathrm{H}$ & $M X, P$ \\
\hline \multicolumn{3}{|l|}{ LYTRACEAE } \\
\hline Heimia salicifolia Link (SR 64, 210, 324) & Ar & MX \\
\hline Punica granatum L. * (SR 356) & $\mathrm{Ar}$ & \\
\hline \multicolumn{3}{|l|}{ MALPIGHIACEAE } \\
\hline Galphimia glauca Cav. (SR 432) & Ar & BE, BTC, MX, P \\
\hline Gaudichaudia cynanchoides Kunth (SR 444, 510) & $\operatorname{Ar}$ & $B C, B Q, M X, P$ \\
\hline \multicolumn{3}{|l|}{ MALVACEAE } \\
\hline Abutilon dugesii S.Watson (SR 349) & Ar & BTC, MX \\
\hline
\end{tabular}


Apéndice. Continuación

\begin{tabular}{|c|c|c|}
\hline TAXA & FC & TV \\
\hline A. hypoleucum A.Gray (SR 77) & $\operatorname{Ar}$ & BTC, MX \\
\hline A. percaudatum Hochr. (SR 256) & $\mathrm{Ar}$ & BTC, MX \\
\hline Allowissadula pringlei (Rose) D.M.Bates (SR 361) & Ar & MX \\
\hline A. racemosa (Schltdl.) Fryxell (SR 248) & Ar & $\mathrm{MX}$ \\
\hline A. sessei (Lag.) D.M.Bates (SR 211) & $\mathrm{Ar}$ & BTC, MX \\
\hline Anoda crenatiflora Ortega (SR 438) & $\operatorname{Ar}$ & BTC, MX \\
\hline A. maculata Fryxell (SR 535) & $\mathrm{H}$ & BTC, MX \\
\hline Bastardia bivalvis (Cav.) Kunth ex Griseb. (SR 273) & $\operatorname{Ar}$ & BTC, MX \\
\hline Herissantia crispa (L.) Brizicky (SR 13, 191) & $\mathrm{H}$ & MX \\
\hline Hermannia pauciflora S.Watson (SR 216) & $\mathrm{Ar}$ & MX \\
\hline Hibiscus elegans Standl. (SR 106, 171) & $\operatorname{Ar}$ & MX \\
\hline Kearnemalvastrum lacteum (Aiton) D.M.Bates (SR 375) & $\mathrm{Ar}$ & $B Q, M X, P$ \\
\hline Malva parviflora L. (SR 332) & $\mathrm{H}$ & BQ, BTC, MX \\
\hline Malvastrum coromandelianum (L.) Garcke (SR 205) & $\mathrm{H}$ & MX \\
\hline Malvaviscus arboreus Cav. (SR 456) & A & MX \\
\hline Pseudabutilon ellipticum (Schltdl.) Fryxell (SR 269) & $\operatorname{Ar}$ & BMM, BTC, MX \\
\hline Sida abutifolia Mill. (SR 37, 47) & $\mathrm{H}$ & MX \\
\hline S. ciliaris L. (SR 259) & $\mathrm{H}$ & BTC, MX \\
\hline S. glabra Mill. (SR 191) & $\mathrm{H}$ & $\mathrm{BMM}, \mathrm{BQ}, \mathrm{BTS}$ \\
\hline S. glutinosa Comm. ex Cav. (SR 275) & $\mathrm{Ar}$ & MX \\
\hline S. linearis Cav. (SR 232) & $\mathrm{H}$ & $\mathrm{BC}, \mathrm{BQ}, \mathrm{BTC}, \mathrm{MX}$ \\
\hline S. procumbens Sw. (SR 100) & $\mathrm{H}$ & BTC, MX \\
\hline S. rhombifolia L. (SR 348) & $\operatorname{Ar}$ & MX \\
\hline Sphaeralcea angustifolia (Cav.) G.Don (SR 298) & $\mathrm{H}$ & MX \\
\hline Triumfetta semitriloba Jacq. (SR 475) & $\mathrm{Ar}$ & BTC, $M X, P$ \\
\hline \multicolumn{3}{|l|}{ MARTYNIACEAE } \\
\hline $\begin{array}{l}\text { Proboscidea louisiana (Mill.) Thell. subsp. fragrans (Lindl.) } \\
\text { Bretting (SR 183, 286) }\end{array}$ & $\mathrm{H}$ & MX \\
\hline \multicolumn{3}{|l|}{ MELIACEAE } \\
\hline Melia azedarach L. * (SR 376) & A & \\
\hline \multicolumn{3}{|l|}{ MOLLUGINACEAE } \\
\hline Mollugo verticillata L. * (SR 549) & $\mathrm{H}$ & \\
\hline \multicolumn{3}{|l|}{ MYRTACEAE } \\
\hline Psidium friedrichsthalianum (O.Berg) Nied.* (SR 371) & A & \\
\hline \multicolumn{3}{|l|}{ NYCTAGINACEAE } \\
\hline Allionia choisyi Standl. (SR 120, 162, 312, 572) & $\mathrm{H}$ & $M X, P$ \\
\hline Boerhavia coccinea Mill. (SR 213, 415B) & $\mathrm{H}$ & BTC, MX, P \\
\hline B. diffusa L. (SR 81, 217, 320, 412) & $\mathrm{H}$ & MX \\
\hline B. erecta L. (SR 207, 310, 415) & $\mathrm{H}$ & BTC, BTS \\
\hline Commicarpus scandens (L.) Standl. (SR 241, 366, 383, 568) & $\mathrm{T}$ & MX \\
\hline Mirabilis glabrifolia (Ortega) I.M.Johnst. (SR 334) & $\mathrm{H}$ & MX \\
\hline M. jalapa L. (SR 452) & $\mathrm{H}$ & MX \\
\hline M. longiflora L. (SR 382) & $\mathrm{H}$ & $M X, P$ \\
\hline M. viscosa Cav. (SR 112B, 212) & $\operatorname{Ar}$ & BTC, MX \\
\hline Salpianthus purpurascens (Cav. ex Lag.) Hook. \& Arn. (SR 463) & $\mathrm{H}$ & MX \\
\hline \multicolumn{3}{|l|}{ OLEACEAE } \\
\hline Forestiera phillyreoides (Benth.) Torr. (SR 583) & A & $\mathrm{BC}, \mathrm{MX}, \mathrm{P}$ \\
\hline
\end{tabular}


Apéndice. Continuación

\begin{tabular}{lll}
\hline TAXA & FC \\
\hline
\end{tabular}

Menodora coulteri A.Gray (SR 234)

M. helianthemoides Bonpl. (SR 168)

$\begin{array}{cc}\text { FC } & \text { TV } \\ \mathrm{H} & \mathrm{MX} \\ \mathrm{Ar} & \mathrm{MX}, \mathrm{P} \\ \mathrm{H} & \\ \mathrm{H} & \mathrm{BQ}, \mathrm{BTC}, \mathrm{MX} \\ \mathrm{H} & \mathrm{BC}, \mathrm{BMM}, \mathrm{BQ}, \mathrm{MX}, \mathrm{P} \\ \mathrm{H} & \mathrm{MX}, \mathrm{P} \\ & \mathrm{BC}, \mathrm{BQ}, \mathrm{MX} \\ \mathrm{H} & \\ \mathrm{P} & \mathrm{BC}, \mathrm{BQ}, \mathrm{MX}, \mathrm{P} \\ & \mathrm{BTC} \\ \mathrm{H} & \\ \mathrm{H} & \mathrm{BQ}, \mathrm{P} \\ \mathrm{H} & \mathrm{BQ}, \mathrm{MX}, \mathrm{P} \\ & \mathrm{BQ}, \mathrm{P} \\ \mathrm{H} & \mathrm{MX}\end{array}$

\section{ONAGRACEAE}

Lopezia miniata Lag. ex DC. (SR 327)

L. racemosa Cav. subsp. racemosa (SR 577)

Oenothera kunthiana (Spach) Munz (SR 74)

O. rosea L'Hér. ex Aiton (SR 448)

\section{OROBANCHACEAE}

Castilleja lithospermoides Kunth (SR 21)

Orobanche dugesii (S.Wats.) Munz (SR 447)

\section{OXALIDACEAE}

Oxalis corniculata L. (SR 499)

O. decaphylla Kunth (SR 397)

O. divergens Benth. ex Lindl. (SR 477)

\section{PAPAVERACEAE}

Argemone ochroleuca Sweet var. ochroleuca (SR 22)

\section{PASSIFLORACEAE}

Passiflora subpeltata Ortega (SR 225)

P. pilosa Ruiz \& Pav. ex DC. (SR 350)

Turnera diffusa Willd. ex Schult. (SR 102)

$\mathrm{T}$

T

$\operatorname{Ar}$

\section{PHYTOLACCACEAE}

Rivina humilis L. (SR 422)

\section{PLANTAGINACEAE}

Bacopa monnieri (L.) Wettst. (SR 208)

B. repens (Sw.) Wettst. * (SR 363)

Maurandya antirrhiniflora Humb. \& Bonpl. ex Willd. subsp. antirrhiniflora (SR 321)

Mecardonia procumbens (Mill.) Small (SR 546)

Russelia equisetiformis Schltdl. \& Cham. (SR 123, 313)

Veronica polita Fr. (SR 69)

\section{PLUMBAGINACEAE}

Plumbago pulchella Boiss. (SR 14, 51, 248, 357, 552)

\section{POLEMONIACEAE}

Loeselia coerulea (Cav.) G.Don (SR 20)

L. mexicana (Lam.) Brand (SR 19, 542)

\section{POLYGALACEAE}

Polygala compacta Rose (SR 48, 113, 153, 316, 537)

P. myrtilloides Willd. (SR 15, 59)

\section{POLYGONACEAE}

Polygonum segetum Kunth (SR 220)

Rumex crispus L. * (SR 579)

R. mexicanus Meisn. (SR 370)
BC, BMM, BTC $\mathrm{BC}, \mathrm{BQ}, \mathrm{BTC}, \mathrm{MX}, \mathrm{P}$ $M X, P$

BQ, BTC, BTS, MX

$B E, B C, B Q, M X, P, V A$

MX, P

VA

VA

$\mathrm{P}$

$M X, P$

$M X, P$

$B C, B Q, M X, P$

$M X, P$

$B E, M X, P$

VA

MX, P 
Apéndice. Continuación

TAXA FC

PORTULACACEAE

Portulaca oleracea L. (SR 73, 209)

P. pilosa L. (SR 333, 480, 516)

\section{RESEDACEAE}

Reseda luteola L. * (SR 140)

$\mathrm{H}$

\section{RHAMNACEAE}

Colubrina elliptica (Sw.) Brizicky \& W.L.Stern (SR 169)

C. heteroneura (Griseb.) Standl. (SR 78)

Condalia mexicana Schltdl. (SR 58)

Karwinskia humboldtiana (Schult.) Zucc. (SR 8)

Ziziphus amole (Sessé \& Moc.) M.C.Johnst. (SR 343, 368)

\section{RUBIACEAE}

Bouvardia ternifolia (Cav.) Schltdl. (SR 17)

Crusea diversifolia (Kunth) W.R.Anderson (SR 75)

Galium mexicanum Kunth (SR 267, 519)

Machaonia coulteri (Hook. f. ex Benth. \& Hook.) Standl. (SR 109, 317)

Randia watsonii B.L.Rob. (SR 287)

\section{RUTACEAE}

Casimiroa edulis La Llave \& Lex. * (SR 558)

Esenbeckia berlandieri Baill. (SR 221)

Helietta parvifolia (A.Gray) Benth. (SR 559)

Zanthoxylum fagara (L.) Sarg. (SR 104, 423)

\section{SALICACEAE}

Salix bonplandiana Kunth (SR 377)

S. taxifolia Kunth (SR 227)

\section{SANTALACEAE}

Phoradendron brachystachyum (DC.) Eichler (SR 49, 429)

\section{SAPINDACEAE}

Cardiospermum halicacabum L. (SR 34, 135, 253, 509)

Dodonaea viscosa Jacq. (SR 393)

Sapindus saponaria L. (SR 462)

\section{SCROPHULARIACEAE}

Buddleia cordata Kunth subsp. cordata (SR 458)

Leucophyllum ambiguum Bonpl. (SR 586)

Verbascum virgatum Stokes * (SR 569)

\section{SOLANACEAE}

Capsicum annuum L. var. annuum * (SR 345)

Datura inoxia Mill. (SR 409B)

D. quercifolia Kunth (SR 538)

D. stramonium L. * (SR 409)

Jaltomata procumbens (Cav.) J.L.Gentry (SR 413)

Lycopersicon esculentum Mill. var. esculentum * (SR 145)

Nicotiana glauca Graham * (SR 18)

N. plumbaginifolia Viv. (SR 139)
A

A

$\mathrm{Ar}$

A

$\mathrm{Ar}$

$\mathrm{Ar}$

A

Ar

$\mathrm{H}$

$\mathrm{H}$

Ar

A

A

A

A

A

$\mathrm{P}$

$\mathrm{T}$

Ar

A

A

Ar

$\mathrm{H}$

$\mathrm{H}$

$\mathrm{Ar}$

Ar

$\mathrm{H}$

$\mathrm{H}$

$\mathrm{H}$

Ar

$\mathrm{H}$
$M X, P$

TV

$P$

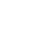

r

$M X$ $B Q, M X, P$ $M X, P$ BQ, BTC, MX, P $M X, P$

$B E, M X, P$ $B Q, M X, P$ $B C, M X, P$ BC, BMM, MX, P $M X, P$

$B E, M X, P$

$B E, M X$ BC, BQ, BTC, BTS, P

$B C, B Q, V A$
$B C, B Q, V A$

BC, BE, BMM, BQ, MX

$M X, P$ $B Q, M X, P$ $\mathrm{BC}, \mathrm{BQ}$

$B Q, M X, P$ $M X$ $M X$ MX $\mathrm{BC}, \mathrm{BQ}$ $B Q, M X, P$ 
Apéndice. Continuación

\begin{tabular}{|c|c|c|}
\hline TAXA & FC & TV \\
\hline $\begin{array}{l}\text { Physalis cinerascens (Dunal) Hitchc. var cinerascens } \\
\quad(\text { SR } 115,132,311,390)\end{array}$ & $\mathrm{H}$ & MX \\
\hline P. patula Mill. (SR 545) & $\mathrm{H}$ & MX \\
\hline P. philadelphica Lam. (SR 315) & $\mathrm{H}$ & $M X, P$ \\
\hline P. sulphurea (Fernald) Waterf. (SR 529) & $\mathrm{H}$ & MX \\
\hline Solanum cardiophyllum Lindl. (SR 301) & $\mathrm{H}$ & $M X, P$ \\
\hline S. elaeagnifolium Cav. (SR 7, 128) & $\mathrm{H}$ & $M X, P$ \\
\hline S. heterodoxum Dunal (SR 296) & $\mathrm{H}$ & $M X, P$ \\
\hline S. marginatum L. f. (SR 159) & $\operatorname{Ar}$ & MX \\
\hline S. umbellatum Mill. (SR 367, 404, 466) & $\mathrm{H}$ & $\mathrm{BE}, \mathrm{BQ}, \mathrm{BTC}$ \\
\hline Witheringia stramoniifolia Kunth (SR 70) & $\mathrm{Ar}$ & $M X, P$ \\
\hline \multicolumn{3}{|l|}{ TALINACEAE } \\
\hline Talinum aurantiacum Engelm. (SR 73) & $\mathrm{H}$ & MX \\
\hline T. paniculatum (Jacq.) Gaertn. (SR 209) & $\mathrm{H}$ & MX \\
\hline \multicolumn{3}{|l|}{ VERBENACEAE } \\
\hline Aloysia gratissima (Gillies \& Hook.) Tronc. (SR 507) & Ar & $\mathrm{MX}$ \\
\hline Lantana camara L. (SR 414B) & $\operatorname{Ar}$ & $\mathrm{BE}, \mathrm{BTC}, \mathrm{MX}, \mathrm{P}$ \\
\hline L. hirta Graham (SR 88) & Ar & $M X, P$ \\
\hline L. velutina M.Martens \& Galeotti (SR 10) & Ar & $M X, P$ \\
\hline Lippia graveolens Kunth (SR 501) & Ar & BTC, MX \\
\hline L. mexicana G.L.Nesom (SR 405) & Ar & BC, BQ \\
\hline Priva lappulacea (L.) Pers. (SR 271) & $\mathrm{H}$ & $M X$ \\
\hline P. mexicana (L.) Pers. (SR 152) & $\mathrm{H}$ & $M X, P$ \\
\hline Verbena gracilis Desf. (SR 147) & $\mathrm{H}$ & $M X, P$ \\
\hline V. recta Kunth (SR 95) & $\mathrm{H}$ & $\mathrm{BC}, \mathrm{BQ}$ \\
\hline \multicolumn{3}{|l|}{ VITACEAE } \\
\hline Cissus verticillata (L.) Nicolson \& C.E.Jarvis (SR 353) & $\mathrm{T}$ & $\mathrm{BC}, \mathrm{BQ}, \mathrm{MX}, \mathrm{P}$ \\
\hline \multicolumn{3}{|l|}{ ZYGOPHYLLACEAE } \\
\hline Tribulus terrestris L. * (SR 231) & $\mathrm{H}$ & \\
\hline
\end{tabular}

